\title{
A Survey of the State-of-the-Art Localisation Techniques and Their Potentials for Autonomous Vehicle Applications
}

\author{
Sampo Kuutti, Saber Fallah, Konstantinos Katsaros, Mehrdad Dianati, Francis Mccullough, Alexandros Mouzakitis
}

\begin{abstract}
For an autonomous vehicle to operate safely and effectively, an accurate and robust localisation system is essential. While there are a variety of vehicle localisation techniques in literature, there is a lack of effort in comparing these techniques and identifying their potentials and limitations for autonomous vehicle applications. Hence, this paper evaluates the state-of-the-art vehicle localisation techniques and investigates their applicability on autonomous vehicles. The analysis starts with discussing the techniques which merely use the information obtained from on-board vehicle sensors. It is shown that although some techniques can achieve the accuracy required for autonomous driving but suffer from the high cost of the sensors and also sensor performance limitations in different driving scenarios (e.g. cornering, intersections) and different environmental conditions (e.g. darkness, snow). The paper continues the analysis with considering the techniques which benefit from off-board information obtained from V2X communication channels, in addition to vehicle sensory information. The analysis shows that augmenting off-board information to sensory information has potential to design low-cost localisation systems with high accuracy and robustness however their performance depends on penetration rate of nearby connected vehicles or infrastructure and the quality of network service.
\end{abstract}

\section{INTRODUCTION}

Between 2000 and 2014 the number of registered road vehicles in the U.S. has increased by 35 million [1], an increase of $15 \%$. This rapid increasing number of road vehicles has amplified the importance of issues such as traffic congestion, pollution, and road accidents. Autonomous vehicles considered as a potential solution to some of these problems by providing more efficient driving and proper vehicle control [2].

The architecture through which autonomous driving is achieved can be described by five functional systems, including Localisation, Perception, Planning, Control, and System Management [3]. The localisation system identifies the location of the vehicle on a global coordinate system while the perception system evaluates the driving environment around

This work was supported by Jaguar Land Rover and the UK-EPSRC grant EP/N01300X/1 as part of the jointly funded Towards Autonomy: Smart and Connected Control (TASCC) Programme.

Sampo Kuutti and Saber Fallah are with the Centre for Automotive Engineering, University of Surrey, Guildford, GU2 7XH, U.K. (e-mail: s.j.kuutti@surrey.ac.uk, s.fallah@surrey.ac.uk).

Konstantinos Katsaros is with Digital Catapult, London, NW1 2RA (email: kostas.katsaros@digicatapult.org.uk)

Mehrdad Dianati is with Warwick Manufacturing Group, University of Warwick Coventry CV4 7AL (e-mail:M.Dianati@warwick.ac.uk)

Francis Mccullough and Alexandros Mouzakitis are with Jaguar Land Rover Limited (fmccull2@jaguarlandrover.com)

Copyright (c) 2012 IEEE. Personal use of this material is permitted. However, permission to use this material for any other purposes must be obtained from the IEEE by sending a request to pubs-permissions@ieee.org. the vehicle and identifies elements such as other road users, traffic signals and obstacles. The planning system uses the inputs from the perception and localisation systems to determine the travel paths and driving actions such as lane changes, accelerating and braking. The control system transforms the behaviour and actions indicated by the planning system to the vehicle manipulation commands (e.g. steering, accelerating and braking). The system management oversees the operation of all the systems and provides the Human-Machine Interface (HMI). These functional systems must cooperate to answer the three important questions of "Where is the car?", "What is around the car?", and "What does the car need to do next?" to achieve fully autonomous operation.

The perception, planning, and control functional systems require accurate knowledge of the vehicle's location to carry out the correct driving decisions and actions. For example, an error of few decimetres can cause the vehicle to localise itself on the wrong side of the road or can cause accidents to the vulnerable users such as pedestrians and cyclists. Robustness is also required as the vehicle needs to localise itself in uncertain driving conditions (e.g. in cases where road markings are absent or obscured) and in harsh environmental conditions (e.g. darkness and snow). Therefore, autonomous vehicles require robust localisation systems with centimetre level of accuracy [4]. The Global Positioning System (GPS) is the most commonly used localisation system for vehicle applications, as they offer a cheap and easily accessible solution for global positioning. However, GPS suffers from poor reliability due to multiple limitations such as signal blockage and multipath as well as inadequate accuracy $(\sim 10 \mathrm{~m})$ for autonomous vehicles. In order to develop robust, accurate, and reliable localisation systems for autonomous vehicle applications, recent research has focused on the development of localisation systems either using advanced sensors (e.g. Radar, LiDAR, camera, etc.) or fusing on-board and off-board information.

Advanced sensors can offer more accurate and reliable position measurements than GPS but at an increased cost. These sensors typically operate by sensing information about the environment, such as road marks, obstacles, other road users etc. However, their performance is limited due to direct line of sight and lack of robustness against harsh environmental situations (e.g. snow, darkness, etc.). Therefore, any obstacle which is obscured cannot be identified, thereby degrading the real-time system performance. Moreover, the cost-effectiveness is a further drawback of these advanced sensors, in order to satisfy the high accuracy requirements of autonomous vehicles. However, with the emergence of Internet of Things (IoT) [5], it is possible for a vehicle to access to a wider range of information (e.g. states of nearby vehicles, 
weather information, traffic status, etc.). By connecting vehicles to inter-vehicle network, intra-vehicle network, and vehicular mobile internet, an IoT infrastructure application can be formed which is referred to as Internet of Vehicles (IoV) [6]. Using IoV, it is possible to enable the next generation of intelligent transportation systems (ITSs) [7], [8], [9]. Providing wireless connectivity to vehicles enables communication with internal and external environments, supporting vehicle-to-vehicle (V2V), vehicle-to-infrastructure (V2I), vehicle-to-sensor, and vehicle-to-internet communications [10]. Such information can be integrated to localisation systems to further improve both accuracy and robustness and to address the line of sight issues associated with on-board sensors at a relatively low cost. The localisation systems which benefit from off-board information are known as cooperative localisation systems. It is noted that in the context of cooperative localisation systems, off-board information can be received either from nearby vehicles, fixed roadside infrastructure units, or both.

There are various research works in the literature proposing different localisation techniques with different approaches in sensor configuration, data fusion methodologies, connectivity, and feature map creation. However, there is a lack of analysing and comparison of these different approaches to identify their potentials and limitations which can be highly beneficial towards deployment of autonomous cars to the market. Therefore, this report aims to survey the state-of-the-art localisation techniques and analyse their performance. To this end, the state of the current field is evaluated and recommendations for future research in vehicle localisation systems are made.

The remainder of the paper is structured as follows. Section II describes mapping techniques used in vehicle localisation context. Section III discusses recent localisation techniques, which utilise only on-board sensors while Section IV is devoted to analysing cooperative localisation techniques. Finally, Section V concludes the current state of the localisation techniques and technologies and makes recommendation for the direction of future work in the field.

\section{MAPPING TECHNIQUES}

Localisation is in principal the process of positioning an object, in the context of this report a vehicle, a pedestrian or other road furniture, with respect to a reference global or local map. In this sense, a proper definition of alternative map types used for vehicle localisation is required. This section defines types of maps relevant to vehicle localisation which are exploited by localisation techniques described in the following sections. In general, there are two main categories of maps: (i) planar which refers to maps that rely on layers or planes on a Geographic Information System (GIS), e.g. High Definition (HD) maps, and (ii) point-cloud which refers to maps based on a set of data points in the GIS. The generation of such maps from companies such as HERE, Google, Uber and TomTom, is challenging and pertains initial data collection, data analysis, and continuous maintenance. Such maps can be maintained exploiting near-real time crowdsourcing information from vehicles travelling and updating backend map systems, e.g. Mobileye's Road Experience Management system [11]. The main difference in using these two types of maps is the type of sensor responsible for localising the vehicle. Vision-based sensors such as cameras, Light Detection and Ranging (LiDAR), Radio Detection and Ranging (RADAR), and ultrasonic use primarily point-cloud maps, whereas GPS-based systems use planar maps. Map matching, i.e. the process of localising the vehicle with respect to the underlying map, is more complex in point cloud systems as it requires intense computations, both at the sensor itself (see next section for details) and for the identification of the points. For that reason, systems such as the NVIDIA DRIVE PX2 [12] introduce high computation capabilities on-board the vehicle. Nevertheless, there is no clear advantage of one type over the other in terms of accuracy of the localisation as the generation of HD/3D-maps entail usage of vision-based sensors during the collection phase.

Planar maps are generated by capturing and analysing data from high-resolution satellite or aerial photography, GPS traces and imaging. The process of detailed recording the environment for later modelling is called micro-mapping in general [13]. The resolution of those maps depends on the captured data and enrichment with additional layers of information from analysed data. For example, HD maps provide a base map layer enriched with a precise sub-lane level representation of the road network, including the lane markings, road furniture, and curvature. With additional details from stereoscopic cameras, three-dimensional (3D) maps provide also the height of objects on the map. In addition to static information about the environment topology, dynamic information such as the traffic conditions, events on the road, location of other moving objects can be incorporated into maps. A standard method to represent such information is the Local Dynamic Map (LDM) [14], [15], that contains several layers of information. Such dynamic information can be used to self-adapt localisation and makes maintenance of accurate map more efficient. For instance, Figure 1 illustrates different layers of a LDM which can be used for the localisation systems of an autonomous vehicle. The first layer represents the static elements of a map, for example, the road network, buildings and other permanent features of a map. This information is usually provided by a map supplier. The second layer contains quasi-static features such as road-side infrastructure, variable speed limits. These are obtained during the operation of the system. The third layer contains transient dynamic data such as weather information, traffic light timings and phase and congestion levels, obtained through interaction with the infrastructure. Finally, the fourth layer of an LDM contains highly dynamic data through Vehicle-to-Everything (V2X) communications and contains location information of other vehicles, Road-side Units (RSU), even pedestrians or cyclists which have such communication capabilities.

On the other hand, point cloud maps are generated by 3D scanners, such as LiDAR sensors, and represent the external surface of an object in the 3D space. The data are stored in $\mathrm{X}$, $\mathrm{Y}$, and $\mathrm{Z}$ format for each co-ordinate where localisation based on point cloud is performed using techniques such as Markov localisation systems and Simultaneous Localisation and Mapping (SLAM) systems [16]. In the former systems, a vehicle collects information about the environment and other road users from on-board sensors and V2X information and uses the information to localise the vehicle inside a pre-existing 
static feature map while, in the latter ones, LDMs are created as the vehicle passes the environment and are then compared to the pre-existing static map to localise the vehicle. The advantage of SLAM is the utilisation of a LDM which means that maps can be updated with each pass of the environment and new maps can be created when the vehicle is in a non-mapped environment. Figure 2 shows an example of cloud point map that can be obtained from a LiDAR, on-board of a vehicle.

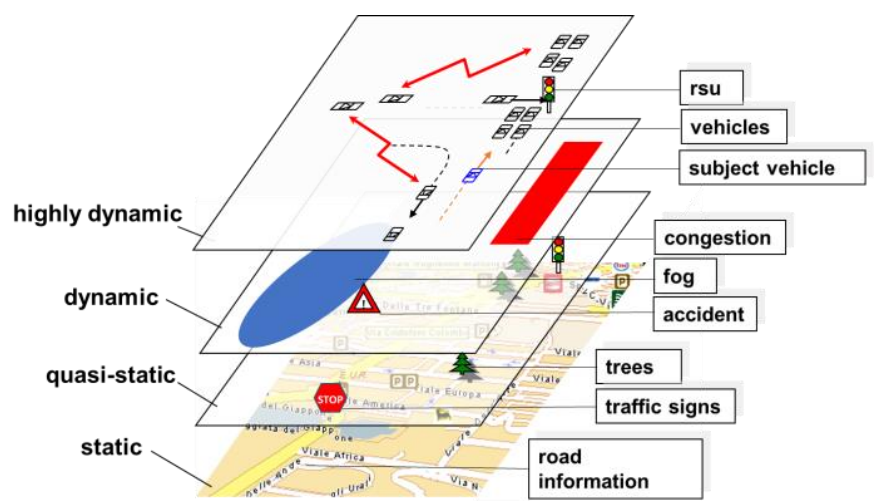

Figure 1. Local Dynamic Map layers with example information. Adapted from [17].

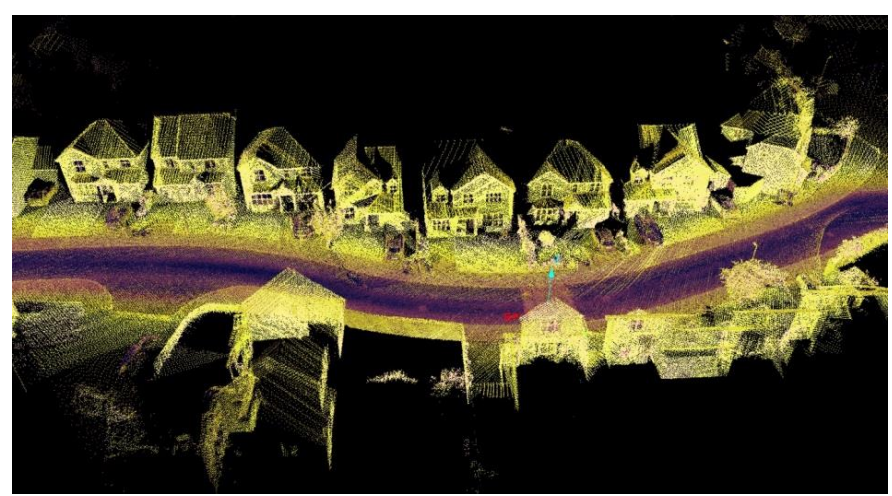

Figure 2. Point cloud captured from LiDAR on-board a vehicle [18].

\section{SENSOR BASED LOCALISATION TECHNIQUES}

These techniques rely only on on-board vehicle sensors to find the global position of a vehicle in a specified coordinate system such as Earth-centred Inertial (ECI) coordinate system, Earth-centred Earth-fixed (ECEF) coordinate system, or the geographical coordinate system. For the brevity of the paper, the detailed definition of each coordinate system is referred to [19]. The main sensors considered in this section include GPS, IMU, cameras, radar, LiDAR, and ultrasonic sensors. The following sections provide details of the capabilities of each sensor including benefits and limitations as well as analysis of localisation techniques using each sensor standalone or a combination of sensors.

\section{A. GPS/IMU Based Techniques}

Global Navigation Satellite Systems (GNSS) such as GPS, GLONASS, BeiDou, and Galileo rely on at least four satellites to estimate global position at a relatively low cost. Typical GPS average accuracy ranges from few meters to above $20 \mathrm{~m}$ [20], limiting its implementation to autonomous vehicle applications as decimetre level $(\sim 30 \mathrm{~cm})$ accuracy is demanded to stay in lane [21]. However, GPS accuracy can be improved upon by using Differential GPS (DGPS), Assisted GPS (AGPS), or Real Time Kinematic (RTK) solutions.

A DGPS utilises measurements from an on-board vehicle GPS unit and from a GPS unit on a fixed infrastructure unit with a known location as shown in Figure 3. As GPS error is correlated between two nearby GPS units, calculating this correlated error can be used to eliminate the error of on-board vehicle GPS to improve its accuracy. DPGS uses the known position of the fixed infrastructure unit to calculate the local error in the GPS position measurement periodically, as illustrated in Figure 3. This correction is then broadcasted to the on-board vehicle GPS units to adjust their own GPS estimate to achieve an average accuracy in the range of 1-2m [19]. Also, AGPS offers increased accuracy and signal availability at an increased cost. AGPS uses information from a cellular network to reduce delays in obtaining a position from the satellite as well as increase the signal coverage by reducing acquisition thresholds [22]. However, it provides less position accuracy compared to a DGPS unit. On the other hand, an RTK-GPS utilises dual-frequency GPS receivers to estimate the relative position of a vehicle with respect to the position of a base station with known position. In this case, the relative position is estimated based on carrier phase measurement of GPS signals to achieve centimetre level accuracy. Further improvements in accuracy could be achieved in the future with the use of the European Galileo GNSS, which is expected to be fully operational with a constellation of 30 satellites by 2020 [23]. The combined GPS-Galileo system is intended to provide centimetre-level accuracy for GNSS solutions with dual-receiver systems [24]. Also, the increased number of available satellites in combined GPS-Galileo systems could improve signal availability.

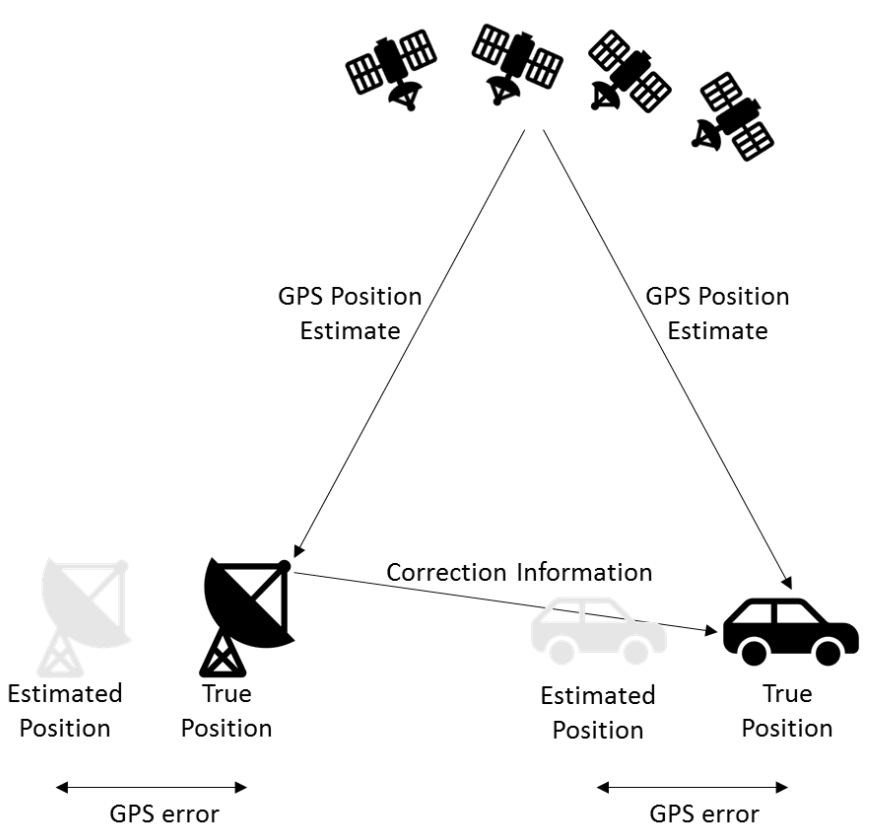

Figure 3. Differential GPS correction. Grey objects indicate estimated position from uncorrected GPS data, while black figures indicate the ground truth. The base station calculates the GPS error from its own GPS measurements and known ground truth, which is then transmitted to the vehicle. 
Although DGPS, AGPS, and RTK-GPS methods improve the accuracy of a conventional GPS, they suffer from poor reliability. Therefore, the signal availability remains a problem, especially in urban environments, thereby reducing implementation potentials of GPS technologies for the autonomous vehicle applications. Additionally, the Time-To-First-Fix (TTFF), which refers to the time required to obtain the signal from the satellites and acquire an initial position estimate after a GPS unit is turned presents another limitation. Typically, TTFF for a GPS unit can be up to 12.5 minutes if the unit was completely turned off. However, this can be partially mitigated by (i) keeping the clock operational whilst the unit is off, (ii) utilising a stand-by mode, or (iii) acquiring the satellite almanac information from a cellular network with AGPS methods [25]. For the aforementioned reasons, GPS needs to be either integrated with other sensors or replaced with more accurate sensors in the context of autonomous vehicles. For this purpose, GPS is often integrated with Inertial Motion Units (IMU) to design a low-cost vehicle localisation system. IMUs use a combination of accelerometers and gyroscopes to measure linear accelerations and vehicle angular velocities, respectively. This information can be used to calculate the trajectory of the vehicle as it travels. For this reason, IMUs have been used to estimate vehicle position relative to its initial position, in a method known as Dead Reckoning. However, the main problem with IMUs is accumulated errors where the measured position drifts further away from its true position as the vehicle travels. This problem can be overcome by correcting the estimated position using other sensors, to avoid accumulated drift and to provide global positioning. To address the low accuracy and interference issues in GPS as well as the cumulative errors related to IMU sensors, the authors in [26] proposed integrating the information from the GPS and IMU. To test the approach, the authors used a GPS/IMU system which provides GPS data, heading angle, and velocity of the vehicle at $10 \mathrm{~Hz}$. The proposed method was successful in increasing the accuracy beyond standalone GPS or IMU capabilities; however cumulative errors were still present in the system. Over a driving distance of $408 \mathrm{~m}$, the system accumulated root mean square (RMS) errors of $7.2 \mathrm{~m}$, compared to that of $22.3 \mathrm{~m}$ and $13.2 \mathrm{~m}$ of IMU odometry and GPS, respectively. Therefore, while this technique was successful in mitigating some of the weaknesses of the standalone GPS and IMU methods, the magnitude of the localisation errors means that the system would be inadequate for autonomous vehicle systems. However, the results show the potential of fusing data from multiple sensors to improve the accuracy and robustness beyond what each sensor can achieve as standalone.

\section{B. Camera Based Techniques}

As a method of replacing GPS with an alternative on-board sensor, the authors in [27] proposed a low-cost localisation method utilising only cameras, where the images obtained from the cameras were down-sampled to a resolution of $800 \times 600$ pixels to reduce computation time. This vision-based localisation approach combines a topological map and a point-cloud map to provide a SLAM type technique. The localisation, first, estimates a rough position through dividing the images into grids and extracting the orientation histograms of each cell. Then, a fine localisation was done using the map consisting of landmarks in the environment. The proposed two-stage localisation method not only increases the accuracy of the localisation but also reduces the computation requirements for the fine localisation. Using the proposed method, mean positioning errors of $75 \mathrm{~cm}$ were achieved. However, the system is sensitive to changes in illumination conditions or angle of observation which may cause the system to fail. Possible solutions to these weaknesses which were suggested by the authors are the use of omnidirectional cameras with a $360^{\circ}$ field of view to mitigate the field of view change errors and designing more robust algorithm against severe weather and illumination conditions. An alternative approach of using cameras is visual odometry discussed in [28], which uses information from cameras to calculate the trajectory of the vehicle for relative positioning. The method suggested by the authors uses stereovision provided by two cameras mounted on the front of a vehicle. The attitude of a vehicle is calculated incrementally by comparing relative movement and orientation to tracked feature points between left and right cameras at different time frames. The algorithm used by the authors is a RAndom SAmple Consensus (RANSAC) algorithm, which rejects outlier estimates to provide robust estimates. Two experiments were completed in an urban environment with cameras recording 30 frames per second at a resolution of $320 \mathrm{x}$ 240 pixels. Using this method, estimated and actual travel lengths of $145.37 \mathrm{~m}$ and $165.86 \mathrm{~m}$ in the first experiment and $197.89 \mathrm{~m}$ and $216.3 \mathrm{~mm}$ in the second experiment were observed, respectively. Main source of errors occurred when the subject vehicle was stationary while other objects moved in the vicinity, therefore potential improvements would be the ability to differentiate between moving and stationary objects. To further improve the performance of the camera-based localisation techniques, the integration of GPS, IMU and camera sensors have been proposed by the researchers. For instance, the authors of [29] suggested using GPS/IMU for global positioning, whilst using the camera to recognise lane markers for lateral positioning. Using this approach, mean positioning errors of $0.73 \mathrm{~m}$ were achieved. The authors of [30] extended the method proposed [29] by the use of cameras for recognition of road markers as well as lane markers to support both lateral and longitudinal positioning. Over two experiments, this approach obtained mean lateral errors of $0.49 \mathrm{~m}$ and $0.58 \mathrm{~m}$ and mean longitudinal errors of $0.95 \mathrm{~m}$ and $1.43 \mathrm{~m}$ for the first and second experiments, respectively. The authors noted that the method has larger longitudinal errors than lateral errors because lanes and road markers were used for lateral localisation, whereas longitudinal localisation only uses road markers. Since lanes are observed with a higher frequency than road markers, the proposed method experiences higher longitudinal errors compared to lateral errors. Major errors were observed (i) when driving on long straight roads with no road markers detected, (ii) when the vehicle was passing through intersections with no road markings or (iii) when false detections of road markers occurred. As another example of integrating vision-based techniques with GPS and IMUs, the authors of [16] proposed two different approaches for localising vehicles based on combination of on-board vehicle camera and aerial images along with IMU/GPS signals. The first method utilises feature maps based on aerial imaging 
containing information about landmarks, lane markings, curbs, and the road geometry. Imaging from the camera equipped on the vehicle was then used to localise the vehicle within the feature map. The second approach uses aerial images which are then processed to remove unnecessary information so that information only about local edges (e.g. the edges of features such as roads) is retained. The experimental results showed that, for the first method, $80 \%$ of position estimates had both lateral and longitudinal errors less than $1 \mathrm{~m}$, while the second method only achieved $60 \%$ of position estimates within this range. Moreover, the second method had peak errors of $3.5 \mathrm{~m}$ and $7 \mathrm{~m}$ which would cause the vehicle to choose the wrong lane. The authors mentioned that future improvements to these techniques could be achieved by further processing of the aerial or camera imaging. Overall, these two methods are still inadequate for autonomous vehicle localisation due to the magnitude of errors present.

In general, although cameras can offer low cost solutions to localisation systems, the level of the accuracy depends on the image resolutions. It is noted that acquiring high resolution images can drastically increases the processing power demand and processing time of the localisation system which cannot be achievable using on-board vehicle processing systems.

\section{Radar Based Techniques}

A Radio detection and ranging (Radar) sensor is a ranging sensor which utilises radio waves. Radar functions by emitting periodic radio waves which can bounce off obstacles back to the receiver and distance to target is measured from the time of arrival of radio waves. Each radio wave provides a single range measurement which gives the distance to the obstacle that reflected it back to the receiver. Radars also have relatively low power consumption, for example the Delphi Short Range Radars use only $0.16 \mathrm{~W}$ and offer up to 64 range measurements at $20 \mathrm{~Hz}$ with a field of view of $\pm 75^{\circ}$ and a range of $80 \mathrm{~m} \mathrm{[31]}$. Even lower power requirements can be achieved by Frequency Modulation Continuous Wave (FMCW) based Radars, which use continuous Radar signals rather than the periodic ones used in traditional Pulse Based Radar systems. For example, the K2pi microwave Radars based on FMCW can offer a $360^{\circ}$ field of view and a range up to $100 \mathrm{~m}$, with power requirements as low as $0.1 \mathrm{~W}$, however the accuracy is typically lower than that of pulse-based radar systems [32].

As an example of localisation system using a Radar, in [32], the authors evaluated the data obtained from a $360^{\circ}$ field of view FCMW microwave Radar sensor through two different SLAM methods to localise a vehicle. The first method is a trajectory-oriented SLAM technique while the other one analyses the distortion caused by rotating Radar at high speed to obtain the trajectory of the vehicle and map the environment. For a vehicle traveling at the speed of $30 \mathrm{~km} / \mathrm{h}$, the methods resulted in mean position errors of $10 \mathrm{~m}$ and $12 \mathrm{~m}$ for the first and second techniques, respectively, thereby indicating the technique will be unsuitable for autonomous vehicles. However, the work in [31] explored the use of pulse-based Short Range Radar (SRR) due to its low cost and good accuracy, where the Radar sensor acquires up to 64 detections, each at $20 \mathrm{~Hz}$. Also, information of speed and yaw rate were used from signals of a GPS/IMU system. The results showed RMS errors of $7.3 \mathrm{~cm}$ laterally and $37.7 \mathrm{~cm}$ longitudinally, with worst case errors of $27.8 \mathrm{~cm}$ laterally and $115.1 \mathrm{~cm}$ longitudinally. While these results are promising for Radar-based localisation, Radar maps are dependent on the quality of discernible features available, which can cause errors when such features are not available. To further improve the accuracies of Radar-based techniques, a novel approach of utilising ground penetrating Radar technology for localisation was proposed in [33]. This method scans the subsurface features and the inhomogeneity of the subterranean geology to create a map. These features are unique and static enough that localisation for autonomous vehicles could be completed utilising subterranean feature maps. Testing was done using a vehicle equipped with GPS/IMU system integrated with the Localising Ground Penetrating Radar (LGPR) system with a ground penetrating depth of $2-3 \mathrm{~m}$, which was brought up to speeds of $100 \mathrm{~km} / \mathrm{h}$ in testing. The vehicles first created a subterranean feature map of the environment over the initial pass of the environment and then attempted to localise itself within this map. Results showed the capability to localise within positional RMS errors of $4 \mathrm{~cm}$, which is within limits for a vehicle to maintain its lane of traffic. The proposed approach, therefore, shows significant potential as it can provide the high accuracy localisation required for autonomous driving. Moreover, unlike localisation systems such as camera-based localisation systems, LGPR is not affected by weather or lighting conditions, thereby making it more robust to different operating conditions. However, the authors pointed out that this concept is relatively new and still requires further study to understand its capabilities and limitations.

\section{LiDAR Based Techniques}

It is noted that Radar maps are susceptible to errors in the case of changes in the pre-existing map due to their limited capabilities in collecting environmental data. Therefore, to increase localisation accuracy and robustness, more accurate maps with denser point clouds are required. LiDAR technology can collect significantly more data than Radar sensors, therefore potentially offer higher accuracy compared to the Radar based techniques. A LiDAR sensor measures distance to a target using multiple laser beams which each measures the distance to the target, based on the time of arrival of the signal back at the receiver, as well as the infrared intensity of the obstacle, as illustrated in Figure 4. LiDAR systems can be used to collect large amounts of ranging and infrared data from obstacles, neighbour road users, etc. For instance, the Velodyne-64 system, which is a common LiDAR sensor used for localisation systems, collects approximately 2.2 million 3D points and their associated infrared intensity values per second over a range of $120 \mathrm{~m}$ and a field of view of $360^{\circ}$ horizontal and $\sim 27^{\circ}$ vertical [34]. However, LiDAR technology is very expensive compared to other sensing technologies and its performance is sensitive to environmental conditions such as rain or snow. The high-power requirement is also a major disadvantage of LiDAR sensors. For example, the Velodyne-64 System uses as much as $60 \mathrm{~W}$ to power itself.

To investigate the use of LiDAR for localisation, the work done in [35] suggested using LiDAR to detect curbs and road markings to create a feature map of the environment and localise vehicles within the map. In the proposed approach, curbs were identified by acquiring LiDAR measurements in 32 
concentric rings and analysing the distance between the rings to identify curb-like features using filters. Also, road markings were identified by analysing the LiDAR reflective intensity data and comparing it to expected values for road markings.

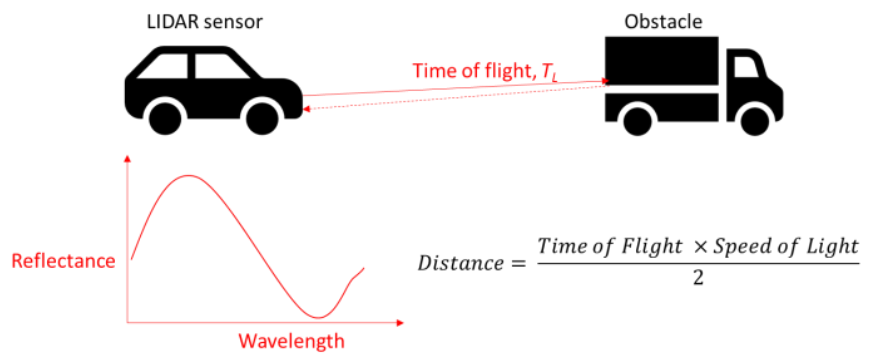

Figure 4. Principle of LiDAR sensor. The sensor sends a laser signal and upon receiving the signal back obtains the infrared reflectivity of the object as well as distance to the object based on time for the signal to bounce back.

These two features were then used to localise the vehicle within the feature map. For the urban experiments, an RTK-GPS, an IMU and a LiDAR sensor with 32 beams and maximum detection distance of $33 \mathrm{~m}$ were used. The proposed approach resulted in lateral and longitudinal errors of less than $30 \mathrm{~cm}$ which were considered satisfactory for autonomous driving in urban environments. In comparison, in [21], the authors proposed a solution integrating GPS, IMU, wheel odometry and LiDAR to generate high-resolution maps. The authors suggested eliminating map features that are unlikely to be static to create a $2 \mathrm{D}$ map of the road surface in the infrared spectrum. Therefore, obstacles such as moving cars are eliminated from the map. A SLAM-style relaxation filter was used to localise the vehicle within the created map at $200 \mathrm{~Hz}$. Using the proposed approach, errors as low as $10 \mathrm{~cm}$ were obtained, although, in some occasions, such as when turning, errors reached as high as $30 \mathrm{~cm}$. The main weakness pointed out in the technique was its reliance on static maps, which meant that extreme changes to the road environments could cause the technique to fail. Possible solutions to this weakness were suggested such as using probabilistic maps to identify changed environments, comparing GPS and map-based localisation to spot localisation errors or creating 3D environment models beyond the road surface. Therefore, to improve robustness to dynamic environments, the technique was further extended in [36] by incorporating the use of LDMs. Instead of creating orthographic maps of the road surface with fixed infrared intensity values, a method of using probabilistic infrared intensity values collected over multiple passes of the same environment was used. The use of probabilistic maps increases robustness to changes in the environment and allows the system to identify dynamic objects. In experimental testing, a GPS/IMU system updating inertial information and global position at $200 \mathrm{~Hz}$ together with a 64-beam LiDAR sensor providing a million $3 \mathrm{D}$ points per second were used. A large amount of acquired data enabled the system to scan map cells multiple times and create a probabilistic map using computation of intensity values. The proposed method was shown to achieve positional RMS error of $9 \mathrm{~cm}$, suggesting accuracy feasible for urban autonomous driving as well as improved robustness to dynamic environments compared to previous methods.
Further improvements to the accuracy of LiDAR based localisation was presented in [37]. The authors utilised an alternative grid representation of the ground which is invariant to the laser-perspective or vehicle motion. This eliminates the need for postfactory reflectivity calibrations required by other methods, improving localisation performance. Using the proposed technique, longitudinal and lateral localisation RMS errors of $3.3 \mathrm{~cm}$ and $1.7 \mathrm{~cm}$ were achieved, thereby improving over the performance of previous state-of-the-art LiDAR localisation methods without increasing the computational requirements. Besides increased accuracy, an additional advantage of using LiDAR is the increased robustness to changes in the mapped environment when compared to techniques such as Radar or camera-based localisation techniques. For instance, in [38] a vehicle equipped with LiDAR, IMU, and wheel odometry successfully drove autonomously in an urban environment. The proposed system localised itself while driving autonomously on a $1.9 \mathrm{~km}$ long route utilising point cloud data collected six months ago and data fusion via a particle filter algorithm. The resulting mean longitudinal and lateral localisation errors were $8.2 \mathrm{~cm}$ and $7.5 \mathrm{~cm}$, respectively. Therefore, highly accurate localisation was still possible even when changes had occurred in the environment and localisation was performed in a different season from the initial mapping data.

While LiDAR techniques can offer very accurate and robust localisation methods, the drawback is their high power and computational requirements as well the high implementation costs. This presents a problem for mass production and marketing of autonomous vehicles equipped with LiDAR sensors. Attempting to reduce the computational requirements of LiDAR based localisation, large point cloud maps were reduced to compact Gaussian mixture maps in [39]. While raw point cloud maps can require up to $500 \mathrm{MB}$ of data per $\mathrm{km}$ of road, the Gaussian mixture maps require 10-100 MB of data, depending on the number of Gaussians per grid cell. Using the Gaussian mixture maps for localisation resulted in longitudinal and lateral RMS errors below $10 \mathrm{~cm}$ and $13 \mathrm{~cm}$, respectively. Moreover, the proposed method was shown to be robust to harsh weather conditions and changes in road appearance due to construction work. On the other hand, addressing the high implementation cost of LiDAR techniques, [40] investigated the use of camera-based localisation within pre-existing LiDAR maps. In contrast to LiDAR, camera-based technology is less accurate and is susceptible to changes in illumination conditions or angle of observation but is significantly cheaper. Therefore, the authors suggested creating initial maps used for localisation using LiDAR sensors and equipping autonomous vehicles with cameras to localise themselves within the LiDAR maps. This means that the highly accurate LiDAR maps are utilised, but autonomous vehicles could be significantly cheaper. This technique was shown to localise with longitudinal and lateral RMS errors of $19.1 \mathrm{~cm}$ and $14.3 \mathrm{~cm}$, respectively, with data captured at $10 \mathrm{~Hz}$, which provides a similar order of magnitude errors to LiDAR techniques but at a significantly reduced cost, power, and processing requirements. Alternative methods of utilising laser technology whilst maintaining low implementation costs, is the use of single beam laser range finders (LRF), such as in [41], where A GPS system, gyroscope, two LRF systems and a 2D feature map, 
consisting of road and building shapes, were integrated. The two LRFs scanned the environment, with one scanning horizontally and one vertically to identify building facades and build a feature map based on this information. Comparing the pre-existing feature map to the local dynamic map resulted in mean positional errors of $3.098 \mathrm{~m}$, which is unsuitable for autonomous vehicle localisation.

\section{E. Ultrasonic Based Techniques}

Other localisation methods have attempted to use alternative low cost sensors such as ultrasonic sensors. For instance, the authors in [42] proposed the use of ultrasonic sensors integrated with a set of sensors including a digital magnetic compass, a gyroscope and two encoders for ultrasonic based SLAM techniques. Ultrasonic sensors can scan the environment by utilising a mechanical wave of oscillating pressure which can propagate through air or other materials. Distance to target can be measured based on the time of arrival of the signals back to the receivers. Ultrasonic sensors were chosen due to their high performance with low electric power consumption and low cost. However, due to inaccurate extracting of feature points, the localisation process could take very long times. Average processing times of $10.65 \mathrm{~s}$ were observed, thereby making the technique unsuitable for high-speed vehicle applications. Also, the associated long average processing time causes accumulated errors due to measurements from the sensors such as IMU. Moreover, the detection range of the ultrasonic sensors is limited to $3 \mathrm{~m}$ which is not sufficient for obstacle detection system of an autonomous system.

\section{F. Discussion}

Table 1 compares the aforementioned localisation techniques in terms of sensor configuration, accuracy, and the associated potentials and limitations. Suitability of the techniques for autonomous vehicles is based on the robustness and reliability as well as capability for in-lane localisation accuracy. The required accuracy for in-lane localisation is taken as $30 \mathrm{~cm}$, as derived in [4]. In summary, the analysis shows that no sensor as standalone offers adequate accuracy and robustness required for autonomous driving and data fusion of multiple sensors has significant potential to design a cost effective localisation system meeting accuracy requirements for autonomous driving. Integrated GPS/IMU/Camera localisation systems provide accuracy up to $73 \mathrm{~cm}$, however further improvements are needed to offer the accuracy and robustness required for fully autonomous vehicles. Radar sensor-based techniques offer cheaper localisation systems compared to LiDAR-based systems and can meet the accuracy requirements for autonomous vehicles, as shown in [33]. However, the robustness of these methods remains an obstacle for implementation. In contrast, LiDAR can offer high accuracy but at a significantly higher cost compared to Radar. Therefore, for LiDAR to be a commercially feasible option further technological advances would be required to reduce the cost or alternatively the approach used in [40] could be used to take advantage of the high accuracy and robustness of LiDAR-based maps, but keep the cost of autonomous vehicles low by equipping them with cameras instead of LiDAR sensors. This type of approach could be the key to achieving high accuracy and formulating low-cost solutions but robust performance in different environment conditions still is a challenge due to limitations of camera systems.

\section{COOPERATIVE LocAlisation TECHNIQUES}

The augmentation of off-board information obtained through V2V and V2I communication systems to the sensory information has shown the potential to improve the vehicle localisation accuracy, robustness, and reliability in different driving and environmental conditions [43]. In such systems, vehicles can broadcast information about their states to other vehicles (V2V), including speed, heading, and location, as well as the information related to the environment while adverse weather conditions or obstacles can be acquired from infrastructure (V2I).

The cooperative localisation techniques use wireless communication devices, such as $\mathrm{Wi}-\mathrm{Fi}$, cellular and UWB radio communications where transmitted signals are used to estimate the range to the broadcaster. There are several approaches to estimate the distance or relative position to the broadcaster of a signal. To calculate the distance to the broadcaster only, one radio signal is required, while calculating a relative position requires three radio signals in case of a two-dimensional localisation or four in the case of three-dimensional localisation. In general, the positioning and ranging systems can work based on four principals, namely time-of-arrival (TOA), time-difference-of-arrival (TDOA), angle-of-arrival (AOA), and radio-signal-strength (RSS). Figure 5 illustrates the concepts of TOA, TDOA, and AOA. In TOA approach, a vehicle initiates the transmission by sending a signal to a reference node, which then sends a signal back, and relative distance to the reference node is measured based on time for the response signal to arrive back at the vehicle. The accurate ranging of TOA approach is limited due to the need of a perfect synchronisation of clocks between all nodes which can be difficult to achieve in practice. In TDOA approach, the position of a vehicle is estimated using transmission signals of three broadcasting stations, where one of the stations, known as the master station, initiates the transmission by sending a signal to the vehicle and the other stations, shown in Figure $5 \mathrm{~b}$ as stations $\mathrm{B}$ and $\mathrm{C}$. Once stations $\mathrm{B}$ and $\mathrm{C}$ receive the initial signal from the master station, they send a signal to the vehicle. Since the location of all stations relative to each other is known, the travel time of radio signals between them is known as well. Based on the time difference of signal arrivals $\mathrm{T}_{\mathrm{AV}}, \mathrm{T}_{\mathrm{BV}}$, and $\mathrm{T}_{\mathrm{CV}}$, the distance to each station and thereby the relative position of the vehicle can be calculated [44]. In AOA approach, as illustrated in Figure 5c, antenna arrays measure the angle of the signal at each node. However, the need for antenna arrays at each node increases the cost and complexity of the system. Finally, in the RSS method, the attenuation of signal strength while traveling from node to node is measured to estimate the travel distance of the signal. It is noted that a radio signal is transmitted from a broadcaster (i.e. a node) which can be stationary (e.g. infrastructure) or mobile (e.g. vehicles).

An Ultra-Wide Band (UWB) sensor uses radio signals to spread information over a wide portion of the frequency spectrum $(>500 \mathrm{MHz})$, allowing large amounts of ranging data to be transmitted with a low power requirement for short distances 
and without interference with other signals in the same spectrum [45]. Moreover, a UWB sensor is capable of penetrating through obstacles due to using the large wavelengths of the radio signals. A UWB sensor estimates the vehicle relative position to a stationary UWB station through communications between different UWB stations. However, the limited range of the signals $(5-50 \mathrm{~m})$ requires well-developed infrastructure thereby limiting the utilisation of UWB sensors for vehicle localisation systems. Therefore, the sensor is typically used in short range, high data rate applications such as indoor industrial autonomous vehicles [46].

a)

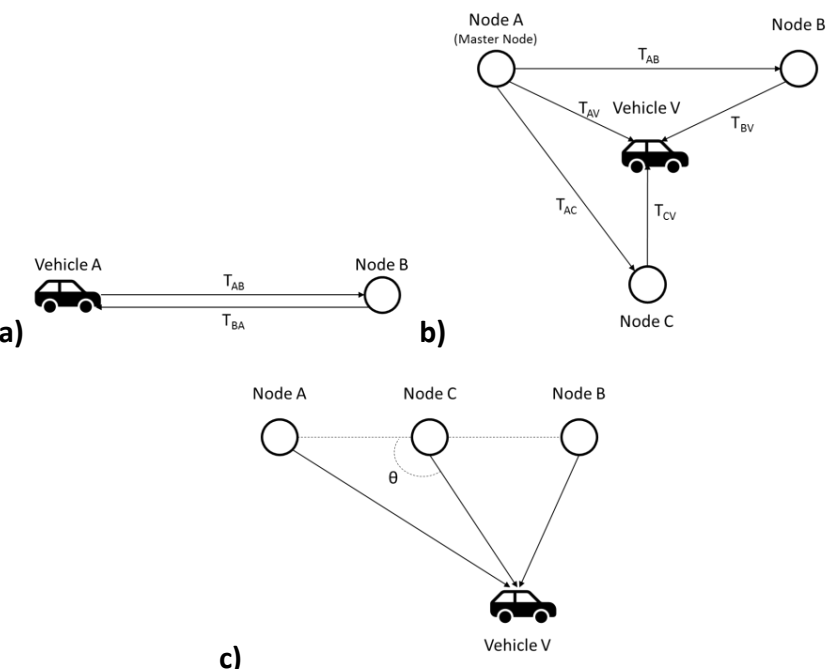

Figure 5. Direction based ranging techniques. a) Time-of-Arrival method b) Time-Difference-of-Arrival method c) Angle-of-Arrival method.

Radio waves can also be used for data communications in wireless networks, such as Wi-Fi or Cellular networks. Wi-Fi devices are defined by the IEEE 802.11 standards, which specify the set of Media Access Control (MAC) and Physical Layer (PHY) specifications that define the Wi-Fi technology. Wi-Fi devices operate in various frequency bands, with a $75 \mathrm{MHz}$ of spectrum in the $5.9 \mathrm{GHz}$ band reserved for Vehicle-to-Vehicle (V2V) and Vehicle-to-Infrastructure (V2I) communications by the European Commission [47]. These devices can be used in vehicle localisation systems to receive information from infrastructure or other vehicles as well as transmit information to other vehicles. The networks can either be infrastructure-based with access points and routers or they can be ad hoc networks in which each node participates in routing by forwarding data to other nodes. Wi-Fi devices achieve data transmission rates ranging from $6 \mathrm{Mbps}$ up to $54 \mathrm{Mbps}$, with communication ranges up to $100 \mathrm{~m}$ and transmission power requirements of up to $100 \mathrm{~mW}$ [48]. On the other hand, cellular networks use technologies such as 3GPP Long Term Evolution (LTE), i.e. 4G, to transfer data between the cellular devices. Cellular networks utilise cellular base stations with a transmission range of up to $15 \mathrm{~km}$ to enable data transmission. Each base station provides communication to a cell around it. As the cellular device travels towards the neighbour cell the signal strength from the next base station becomes stronger and therefore the device switches communications to that base station in a procedure called hand-off. Although cellular devices typically only use one base station for communication, multiple cellular base stations are typically within communication range, which enables localisation through the ranging methods discussed previously. Cellular networks typically broadcast in the 800, 900, 1800, 2100 or $2600 \mathrm{MHz}$ bands, achieving transmission rates up to $100 \mathrm{Mbps}$ [48].

\section{A. Vehicle-to-Vehicle Localisation Techniques}

In this paper, a $\mathrm{V} 2 \mathrm{~V}$ localisation technique refers to a localisation technique which integrates information from adjacent vehicles with the on-board sensory information. V2V communication systems form a Vehicular Ad-Hoc Network (VANET) of interconnected mobile nodes which are aware of the locations and trajectories of each other. Although distance measurements to a VANET broadcaster cannot be as accurate as for example LiDAR measurements, it offers distance measurements out of line-of-sight and at relatively low cost and less processing requirements. $\mathrm{V} 2 \mathrm{~V}$ systems are usually based on the IEEE 802.11p protocol, which is an extension to the IEEE 802.11 protocol that defines the standards for $\mathrm{Wi}-\mathrm{Fi}$ technology. The IEEE 802.11p based Wireless Access in the Vehicular Environment (WAVE) technology can offer bit rates of $3-27 \mathrm{Mbps}$ over a communication range of up to $1000 \mathrm{~m}$ [48]. The message types expected to be used in VANET systems can vary regionally, for example in the European context, V2V communication systems mainly depend on the use of three different types of messages; Cooperative Awareness Messages (CAMs), Decentralised Environmental Notification Messages (DENMs) and Service Announcement Messages (SAMs). CAMs are periodically transmitted to share vehicle information (position, dynamics, attributes etc.) to other road users thereby maintaining cooperative awareness in the VANET [49]. DENMs are event driven messages containing information about an event, such as an accident or a hard-braking vehicle, to other nearby vehicles [50]. SAMs are messages sent by vehicles or infrastructure on the control channel to announce services available on the so-called service channels [51].

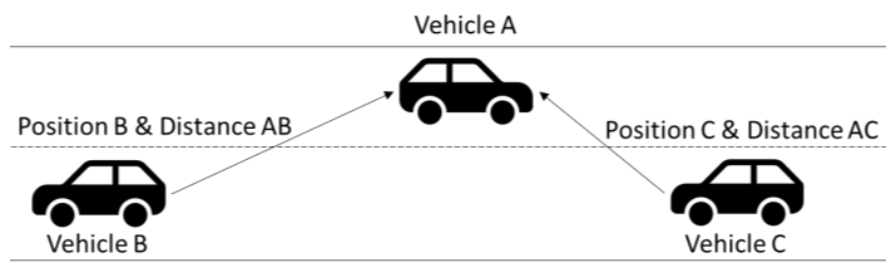

Figure 6. V2V Multilateration. Transmitted positions and calculated distances to the nearby vehicles are used to obtain additional self-localisation estimates for subject vehicle A.

The widely-used technique of V2V systems to improve GPS accuracy is multilateration, illustrated in Figure 6, in which multiple GPS measurements from adjacent vehicles as well as distance to the other vehicles are used to increase the overall accuracy of a vehicle's self-localisation estimate. The more position estimates the vehicles can acquire, the more accurately they can estimate their own position [52]. In [53], simulation of vehicles equipped with multilateration (V2V and GPS) systems was carried out to show that $\mathrm{V} 2 \mathrm{~V}$ communication can reduce average localisation errors from $6.75 \mathrm{~m}$ for GPS-only localisation, down to $3.30 \mathrm{~m}$. However, factors such as uncertainty of inter-vehicle distance, the number of vehicles in 
a cluster, communication latency and communication failures will affect this performance. During communication failures or with high errors in the inter-vehicle distance measurements, these errors tend towards the GPS-only localisation error. Similarly, in [52] the localisation accuracy of a multilateration system was improved by integrating information from on-board sensors, such as millimetre wave Radar. Using simulation results and assuming a communication interval of $100 \mathrm{~ms}$ and a maximum sensing range of $100 \mathrm{~m}$, the authors showed that their approach, when all vehicles were equipped with GPS and V2V communication devices, achieved an average error of $0.6 \mathrm{~m}$. Therefore, the proposed approach reduced the localisation error by $85 \%$ compared to GPS solutions and $60 \%$ compared to a multilateration system. In the research, while the accuracy inter-vehicle distance estimation depends on the number of vehicles with $\mathrm{V} 2 \mathrm{~V}$ communication systems, it was shown that if $100 \%$ of the vehicles are equipped with a V2V technology, the system recognised $70 \%$ of all nearby vehicles within an error of $1 \mathrm{~m}$ while if $40 \%$ of vehicles were equipped with V2V systems, only $50 \%$ of all nearby vehicles within an error of $1 \mathrm{~m}$ were recognised by the system. Therefore, the relationship between the number and penetration rate of connected vehicles and localisation accuracy is non-linear where a minimum number of connected vehicles would be required for adequate accuracy and reliability. Also, the results of the simulations show that the $\mathrm{V} 2 \mathrm{~V}$ localisation paired with on-board ranging sensors can achieve good accuracy. This approach was also evaluated in field tests, in [54], where six vehicles with GPS/IMU and V2V systems were tested with one vehicle with a highly accurate positioning system to be used as the ground truth. Measurements from two of the six vehicles showed unexpected behaviour and were therefore removed from the results. The remaining results showed that $50 \%$ of the errors were within $1.09 \mathrm{~m}$, resulting in an improvement of $66 \%$ compared to GPS as well as $95 \%$ errors were within $3.54 \mathrm{~m}$, resulting in an improvement of $85 \%$ compared to GPS. Therefore, the accuracy of VANET localisation is highly dependent on the number of connected vehicles in the area, as well as the accuracy of the shared position estimates. Moreover, the unexpected behaviour of the two vehicles in the experiment highlighted the need to mitigate the possible effect of erroneous inputs from the other vehicles. Therefore, the localisation algorithm would need fault detection and isolation to reject any erroneous inputs.

The authors in [55] extended multilateration methods to include the use of stationary vehicles for improving the localisation accuracy. Since stationary vehicles can localise themselves more accurately given enough time, the authors propose using stationary vehicles (e.g. parked vehicles) to transmit their location via $\mathrm{V} 2 \mathrm{~V}$, so nearby vehicles can gain a more accurate self-localisation estimate. Although battery discharge of a powered-off vehicle is a concern, the authors pointed out that using a $1 \mathrm{~W}$ on-board unit, a $480 \mathrm{Wh}$ battery can last up to 2 days. The method was evaluated using a small-scale and a large-scale scenario with 20 and 900 cars, respectively. Compared to multilateration without stationary vehicles, the simulation results showed up to $55.09 \%$ and $62.85 \%$ improvement in the small-scale and large-scale cases, under best scenarios with the highest number of parked cars. The average improvement for both test cases was $33.01 \%$. This shows the potential improvement in accuracy from the use of stationary vehicles in V2V systems. Furthermore, in [56] the authors used multiple GPS position estimates from other vehicles and ranging estimates using TOA and AOA measurements to improve localisation accuracy. Also, the proposed approach used vehicle state information from a gas pedal, brake pedal, and steering wheel sensors. In this method, the vehicles used the shared state information from other vehicles to gain a better estimation of their own location as well as estimate the location of the other vehicle and then sent this information back to them. Therefore, vehicles not only gain information regarding other vehicles in the network, they acquire more estimates about their own position as well. The proposed approach was tested in simulations with 5 vehicles and achieved a mean square error (MSE) of $0.52 \mathrm{~m}$ for the best performing vehicle and $1.65 \mathrm{~m}$ for the worst one.

Another approach for improving multilateration accuracy is weighted average localisation. This approach assigns confidence values to each position estimate received, and gives higher weight to measurements with higher confidence in the localisation algorithm. For example, in [57], the authors proposed a method with weights determined based on distance to the broadcaster, since vehicles further away from the subject vehicle are more likely to produce erroneous distance measurements. The proposed method was evaluated with a network of 10 cars, resulting in a mean localisation error of $2.38 \mathrm{~m}$. This approach was further extended in [58] to include Signal to Noise Ratio (SNR) in the determination of weight factors as well, since signal noise can affect the localisation accuracy. The approach was evaluated through multiple simulations with network sizes ranging from 20 to 200 cars. The localisation errors observed ranged from $85 \mathrm{~cm}$ for 20 cars down to $25 \mathrm{~cm}$ for 200 cars. This shows that the inclusion of confidence values in a multilateration system can greatly improve accuracy.

It is important to note that all abovementioned cooperative localisation techniques rely on the use of GPS for the vehicle self-localisation. Whilst GPS may give adequate performance in most scenarios, as mentioned before, GPS suffers from poor reliability in some environments such as dense urban environments. Therefore, in safety critical applications such as autonomous vehicles, the localisation system would need to be fault tolerant in order to continue operating with adequate performance even in the case of high GPS errors or signal blockage. Simply integrating the GPS with other on-board sensors would be a possible solution to offer reliability through sensor redundancy [59]. Other considerations for the reliability of $\mathrm{V} 2 \mathrm{~V}$ localisation systems are the network quality of service. In [47] it was noted that the limits of the $802.11 \mathrm{p}$ can act as a barrier to situational awareness since the $5.9 \mathrm{GHz}$ band used is very sensitive to obstacles. While in good communication condition the authors could establish a link from 700m, a single thin wall of trees could create as much as $25 \%$ packet loss with distances as small as $50 \mathrm{~m}$. Therefore, the effect of highly congested networks with fast moving nodes and varying topology on the performance on cooperative localisation systems will need to be considered.

Table 2 shows a summary of the V2V localisation methods discussed above. Currently, the most researched V2V techniques are multilateration systems which utilise GPS and 
V2V communication. The results in Table 2 show that these techniques can greatly improve GPS localisation accuracy. However, while the accuracy of V2V localisation systems based on multilateration are not adequate for autonomous vehicles, they show great promise for integration to more accurate on-board sensor based techniques (e.g. camera-based or LiDAR based) to further improve performance, accuracy, reliability, and robustness at a relatively low cost. The main limitation of $\mathrm{V} 2 \mathrm{~V}$ localisation techniques is their reliance on high penetration rate and number of connected vehicles which cannot always be guaranteed to be available.

\section{B. Vehicle-to-Infrastructure Localisation Techniques}

In Vehicle-to-Infrastructure (V2I) systems, the vehicles communicate with road side units (RSUs) or cellular base stations at fixed positions. This allows the RSUs to communicate their location, weather conditions, traffic flow, etc. with vehicles to estimate their own position more accurately. The potential advantages of the V2I systems over V2V systems include (i) offering a more accurate location estimate due to fixed position of RSUs (similar to the stationary cars in the V2V localisation, but more reliable as the RSUs are fixed rather than only temporarily stationary), (ii) improved reliability as the number of RSUs within transmission range can be guaranteed unlike in $\mathrm{V} 2 \mathrm{~V}$ techniques, (iii) broadcasting critical information (e.g. events such as accidents which have occurred in the area or adverse weather conditions), allowing the vehicles to have a greater awareness of their environment and allowing them to react to any risks, (iv) enhancing traffic flow and safety through supporting vehicle control and traffic management systems where vehicle speed, steering, and route selection are controlled by a control station aware of other vehicles on the road. However, the drawback of V2I systems based on the IEEE 802.11 p technology is the large number of RSUs required for continuous communication, which will require large initial installation works and costs. Therefore, to limit these initial costs the design of V2I communication systems should be efficient and reliable without needing excessive infrastructure implementation. As such, the spacing and configuration of RSUs must be studied, as well as different communication methods and their effect on system parameters (e.g. networks latency, packet loss, etc.). As shown in [60], output power transmission, antenna height, and tilt, the length of packets and the access mechanism, all affect the performance of V2I communication networks. Also, optimal configurations of roadside beacons may vary under different traffic loads. Further, the use of existing cellular infrastructure can be used in order to reduce capital expenditure.

There are multiple methods for estimating the relative position from the broadcaster of a signal as discussed previously. For instance, [61] proposed the use of Impulse Radio Ultra-Wide Band (IR-UWB) signals for V2I localisation and compared different methods for relative positioning to the broadcaster. The main benefits of IR-UWB are low-transmit power, low cost, wide bandwidth usage, high precision, and resistance to multipath errors due to short pulses. The proposed system uses V2I communication devices placed at lampposts at $20 \mathrm{~m}$ intervals to broadcast their position to nearby vehicles. Based on the distance to the road side units and the distance measured from the RSU, the vehicle can estimate its position. The authors also discussed various methods such as TOA, TDOA, and AOA for estimating the distance between the vehicle and RSUs. In simulations, TDOA was observed to be the most efficient at the middle of the network, whilst AOA was the most efficient at the edges of the network. Therefore, the authors suggest a hybrid technique utilising TDOA and AOA techniques would be optimal for V2I applications.

In contrast, [62] proposed a localisation technique based on AOA estimation. In the proposed system beacon packets from a RSU were used to combine the AOA of the signal and the location of the RSU broadcasted in the signal into a least squares algorithm to localise the vehicle. The system was tested in a simulation and showed very good accuracy when close to the RSU, due to high SNR and favourable geometrical conditions. The performance however degraded as the distance between the vehicle and RSU increased, with GPS-level accuracy obtained at approximately $155 \mathrm{~m}$ away from the RSU. However, with closer spacing of the RSUs, this weakness could be mitigated. The benefit of this technique is that no sensors are required on-board the vehicle apart from V2I communication systems, therefore the main cost of the system would be the implementation of the RSUs. On the other hand, in [63], the authors suggested V2I localisation system for a vehicle equipped with IMU and V2I communications. Localisation is based on two-way TOA ranging with an RSU and vehicle kinematics from the IMU, which achieved RMS errors of $1.8 \mathrm{~m}$. An alternative approach using infrastructure to provide lateral positioning for in-lane localisation was presented in [64], where the authors used passive transponders integrated into lateral white strips on the roads and on-board units to detect the transponders. These passive transponders, unlike the RSUs in other methods, do not broadcast a signal but only reflect the signal from the on-board sensors back to the vehicle. The benefit of the proposed approach is a very low cost of the transponders and large penetration depth in water and snow due to the ultra-high frequency band utilised. The proposed method was tested in several laboratory experiments including in presence of parasitic reflectors, asphalt, and other transponders. The results showed very high accuracy with errors below $3 \mathrm{~cm}$ in all experiments. However, the low range of the utilised method is a drawback; all experiments were completed for distances below $2 \mathrm{~m}$, and the results showed increasing error with distance. Therefore, the transponders would need to be deployed at small intervals and a large quantity of them would be required, although their low cost helps mitigate this drawback. However, further testing would be required in a scenario with the transponders deployed on the road with all the adequate protection required and with moving vehicles to validate these high accuracies and investigate the robustness of the system to more diverse operating conditions.

It is noted that communication systems for advanced driver assistance systems or autonomous vehicles need to have low latency, high accuracy, and strong security. The future fifth-generation (5G) mobile networks are expected to offer increased reliability, reduced latency, and higher throughput compared to current mobile networks, showing great promise for an alternative approach to current vehicle communication systems [4]. The authors in [65] studied the feasibility of 5G networks for positioning using 5G-like multicarrier signals and TDOA measurements. The considered scenario was on a 
highway with base stations deployed at $200 \mathrm{~m}$ intervals. The simulation results showed that at 50 and $100 \mathrm{MHz}$ signal bandwidth the position accuracy was below 25 and $20 \mathrm{~cm}$ in $99 \%$ of the cases, respectively. These results showed that $5 \mathrm{G}$ networks can offer very accurate positioning, adequate for in lane positioning. Moreover, the use of existing infrastructure, such as lampposts on a highway, can reduce the cost of deploying the $5 \mathrm{G}$ base stations.

In general, the main drawback of V2I techniques is the high cost of implementing new dedicated infrastructure. A possible solution to mitigate this problem is utilising existing cellular infrastructure for localisation. A cellular device can generally communicate with multiple cellular base stations at any given time, which enables techniques such as RSS to estimate the position of the phone based on the distance to the base stations. There are two approaches to utilising RSS measurements for cellular-based localisation: triangulation and fingerprinting. Triangulation uses path-loss models to estimate the distance to multiple base stations and then the vehicle location can be calculated based on the location of each base station and the estimated distance to them. However, RSS-based triangulation has been shown to provide limited accuracy due to the inaccuracy of the path-loss models [66]. On the other hand, in fingerprinting the signal characteristics from base stations are recorded at different locations and stored in a database. The experienced signal characteristics can then be compared to the reference values in the database to estimate the position of the cellular device [59]. While fingerprinting methods have been successful in indoor environments with up to $5 \mathrm{~m}$ accuracy [67], average errors up to $94 \mathrm{~m}$ were seen in an outdoor urban testing [68]. Alternatively, fingerprinting could be based on signals from Wi-Fi Access Points, which has been shown to achieve localisation errors of $18 \mathrm{~m}$ and velocity estimation errors of $25 \%$ [69]. However, in [70] a novel approach to fingerprinting was presented in which a vehicle was equipped with 16 GSM devices to scan different GSM channels in parallel, GPS for global positioning, as well as IMU and magnetic compass for trajectory estimation. The RSS values of each channel were then recorded on different roads and combined with the trajectory estimation to localise the vehicle based on these values. The authors utilised a two-stage localisation technique where a coarse localisation was first done based on GPS data, and then a fine localisation based on the GSM RSS characteristics and vehicle trajectory estimation. Average localisation errors of $4.2 \mathrm{~m}$ with $90 \%$ errors within $5.3 \mathrm{~m}$ were achieved using this approach. However, one drawback of the method is the update requirements of the RSS characteristics, as the average localisation error increases to $4.6 \mathrm{~m}$ when using six-week-old data. Therefore, the localisation accuracy could significantly degrade over time if updates are not completed at regular intervals, which would increase the cost of the system. However, this approach would not require new infrastructure, therefore the main associated costs would be equipping the vehicles with the necessary sensors and updating the GSM scan databases.

Techniques integrating V2V, V2I, and on-board sensors have also been investigated by researchers. For instance, in [71], the authors combined V2V/V2I communication with GPS, IMU, magnetic sensor, and laser scanner. The proposed method used magnetic markers positioned at intersections and/or roundabouts, of which the known location was broadcaster by an infrastructure agent to nearby vehicles. Additionally, GPS/IMU was used for global positioning, whilst the laser scanner was used to provide range and direction estimates of the vehicles in front. This technique achieved RMS errors of $1.03 \mathrm{~m}$, which still has room for improvement but shows the potential for the combination of V2V and V2I communications systems for providing low-cost and accurate vehicle self-localisation. Similarly, in [72], V2V/V2I communications were integrated with GPS. In addition to localisation, the authors attempted to provide improved yaw, pitch and roll estimates over traditional GPS/IMU systems. In the proposed architecture, there are three main components: road vehicles, RSUs and master antenna fixed stations (MAFS). The road vehicles are equipped with two GPS receivers positioned longitudinally on the vehicle's roof. The RSU was equipped with a GPS receiver and V2I communication system. The MAFS was equipped with a GPS receiver in a fixed position with good signal connectivity. The MAFS then broadcasts its position to the other units. The system was evaluated using experiments which showed that the system is capable of self-localisation within RMS errors of $0.698 \mathrm{~m}$ and standard deviation of $0.14 \mathrm{~m}$. Moreover, the system showed good performance in Yaw and Pitch estimations, obtaining mean errors of $7.90^{\circ}$ and $0.86^{\circ}$, respectively. However, roll estimates showed large errors, with the mean error of $40.70^{\circ}$. The high roll estimate error is due to roll estimates requiring collinear vehicles that are close to each other, however when the vehicles are nearly collinear the estimates are very sensitive to noise, leading to high errors.

Similarly, combining V2V and V2I communication for localisation, in [73] a localisation technique utilising radio frequency identification (RFID) tags and readers, IMUs, and $\mathrm{V} 2 \mathrm{~V}$ communication was proposed. The proposed method used a matrix-based tag deployment strategy, where tags were deployed in clusters, with multiple adjacent clusters forming rows of tags. Each vehicle was then provided a map of the locations of each RFID tag. The advantage of the proposed method is that accurate localisation could be achieved with low-density and low-cost RFID infrastructure. As a first step, the method was evaluated in a highway simulation with average vehicle speeds of $108 \mathrm{~km} / \mathrm{h}$. It was shown that using inter-tag intervals of $100 \mathrm{~m}, 500 \mathrm{~m}$, and $900 \mathrm{~m}$ resulted in mean absolute errors of $0.5 \mathrm{~m}, 1.4 \mathrm{~m}$, and $2.9 \mathrm{~m}$, respectively. Next, the proposed method was evaluated in experiments with inter-tag intervals of $200 \mathrm{~m}$ and two vehicles driven at speeds of $60 \mathrm{~km} / \mathrm{h}$. Mean absolute errors of $0.71 \mathrm{~m}$ for localisation were achieved, with higher errors observed when the vehicle was turning. Therefore, even with relatively sparsely deployed tags, resulting in a cost of a few dollars per kilometre of road, sub-meter localisation accuracy could be achieved.

Another emergent communication technology is Visible Light Communication (VLC), which has gained interest for V2I [74], [75], [76] and V2V [77], [78], [79], [80] applications. VLC utilises low cost light-emitting diodes (LEDs) to transmit information by turning the LED light on and off at a rate not perceivable to the human eye [81] supporting data rates up to $96 \mathrm{Mbps}$ [82]. This enables transmission of data in V2I application via traffic and street lights with ranges of 50-100m [83] and in V2V applications via headlamp and taillights with 
ranges of 40-50m [80]. VLC has been shown to provide good localisation accuracy in indoor environments, such as Epsilon [84], which utilised RSS and trilateration to provide localisation with $90^{\text {th }}$ percentile accuracy of $0.4 \mathrm{~m}$ in an office environment. However, adapting VLC to outdoor vehicular applications introduces further complications, such as signal attenuation from severe weather conditions and ambient noise from direct sunlight or other strong light sources. Robustness to ambient noise can be improved by utilising higher frequencies and high pass filters. Yu et al. [80] investigated the localisation accuracy of VLC in a V2V setting at multiple signal frequencies through numerical analysis, claiming that accuracies below $1 \mathrm{~m}$ and $0.1 \mathrm{~m}$ could be achieved by headlamps with signal frequencies of $20 \mathrm{MHz}$ and $40 \mathrm{MHz}$, respectively. It should be noted that increasing the signal frequency has the drawback of increasing processing requirements and degrading transmission power. Also, simulation results by Kim and Jung [85] suggested that sub-meter localisation accuracy could be achieved when within $20 \mathrm{~m}$ of LEDs in a tunnel environment where both tunnel ceiling lights and vehicle taillights were utilised for trilateration. However, the main drawback of VLC when compared to DSRC is the direct Line-of-Sight requirement and lower communication range. In an urban scenario simulation, $\mathrm{Yu}$ et al. [80] showed that DSRC could communicate with an average of 32 vehicles, while VLC performed significantly worse by communicating only with an average of 5 vehicles in the same scenario. Nevertheless, VLC could be well suited to some environments such as tunnels or carparks where the outdoor limitations of VLC do not apply as a dense LED deployment can be realised with constant uptime and with little ambient lighting, thereby providing low cost V2X communication with highly accurate localisation capability. Overall, VLC is a promising communication alternative for $\mathrm{V} 2 \mathrm{X}$ applications, but applications to vehicle localisation currently suffer from lack of research.

\section{Discussion}

Management of network traffic and quality of service in both V2V and V2I systems require signals with low latency as low as $10 \mathrm{~ms}$ [4]. Packet loss within the network should be also minimised, therefore the network parameters (e.g. signal bandwidth, transmission power, etc.) need to be optimised for scalability of the networks as interference in congested networks can cause high packet loss ratios and reduce the effective reliable transmission range by up to $90 \%$ [86]. Moreover, in [51], it was noted that while cooperative localisations can perform effectively under high speed and good communication conditions, in poor communication conditions the effectiveness can be significantly reduced. The performance degradations can be overcome by modifying the communication parameters such as transmission power, packet transmission frequency, and data rate. However, this can introduce other problems such as channel congestion. A further limitation of the $\mathrm{V} 2 \mathrm{~V}$ communication systems is discussed in [87], where the applicability of various routing protocols is discussed for VANET applications. The node behaviour in urban and highway environments can vastly differ due to differences in vehicle speed, mobility patterns, and vehicle densities. This means that different routing protocols will have varying levels of performance in urban and highway scenarios; while some are more suited to the urban environments others are more suited to highway environments. Therefore, a hybrid routing protocol may be required that can adapt itself to different environments. Furthermore, the security of the network must also be ensured. Falsified or altered messages or Denial-of-Service attacks could severely impact the safety of VANET applications. Similarly, the privacy of the network needs to be maintained since most drivers will want to maintain the privacy and protection of any information sent over the network to avoid the possibility of their position being tracked. Therefore, a security mechanism for the network will be required to protect the integrity of the network whilst maintaining the users' privacy [88].

A summary of V2I localisation techniques is represented in Table 3. The comparison of the results in Tables 1, 2, and 3 shows that the accuracy of localisation systems highly depends on the information sources utilised. For instance, among the sensor based techniques, LiDAR-based techniques can achieve very high accuracy and robustness at the cost of high power requirement and costly sensor with very limited performance in harsh environment conditions. It was also shown that the methods such as camera-based localisation or LGPR techniques can offer adequate accuracy for autonomous vehicles at a lower cost at the expense of the lack of robustness against variation of environmental conditions. In general, the main limitations of all sensor-based techniques are their limited line of sight, out of range environmental information, and limited operation in harsh environment. V2V techniques have presented great potential to address such limitations of sensor-based techniques. Currently, the only available V2V localisation technique is multilateration which can significantly improve the accuracy of GPS/IMU sensor-based technique when an adequate number of connected vehicles are available. For example, the weighted average localisation in [58] achieved very high accuracy in a network of 200 cars, but the accuracy decreased as the size of the networks decreased. The VANET quality of service also limits the reliability of V2V techniques since network noise will affect the received signals causing erroneous inputs into the localisation algorithm. On the other hand, V2I localisation techniques can mitigate the difficulty of guaranteeing an adequate number of signals and accuracy of broadcasted position estimates in V2V localisation techniques as the RSUs are installed in known fixed positions and the RSU density can be optimised to provide adequate accuracy and robustness. However, V2I techniques require costly infrastructure implementations to ensure high accuracy and robustness. Alternatively, utilising existing cellular networks provides a method for V2I localisation without the need for implementation of new costly infrastructure. Similar to $\mathrm{V} 2 \mathrm{~V}$ techniques, the quality of service in V2I networks is also a limitation for implementation as noise can affect the received signals causing erroneous inputs and packet loss and latency can cause degradation in performance or failure of localisation systems. A summary of localisation techniques considered adequate for autonomous vehicle localisation from a performance point of view is given in Table 4. 
Table 4. Localisation Techniques Adequate for Autonomous Vehicles.

\begin{tabular}{|c|c|c|}
\hline $\begin{array}{l}\text { Technique } \\
\text { (Reference) }\end{array}$ & Sensors & Accuracy \\
\hline $\begin{array}{l}\text { Localising Ground } \\
\text { Penetrating Radar } \\
\text { [33] }\end{array}$ & LGPR, GPS, IMU & $\begin{array}{l}0.04 \mathrm{~m} \\
\text { (RMSE) }\end{array}$ \\
\hline $\begin{array}{l}\text { LiDAR SLAM } \\
\text { [35], [21], [36], [37], } \\
\text { [38], [39] }\end{array}$ & LiDAR, GPS, IMU & $\begin{array}{l}0.017 \mathrm{~m} \text {, lat. } \\
0.033 \mathrm{~m} \text {, long. } \\
\text { (RMSE) }\end{array}$ \\
\hline $\begin{array}{l}\text { Camera localisation } \\
\text { within LiDAR map } \\
\text { [40] }\end{array}$ & Camera, IMU & $\begin{array}{l}0.14 \mathrm{~m} \text {, lat. } \\
0.19 \mathrm{~m} \text {, long. } \\
\text { (RMSE) }\end{array}$ \\
\hline $\begin{array}{l}\text { RF Infrastructure } \\
\text { Localisation } \\
\text { [64] }\end{array}$ & On-board UHF antenna & Up to $0.03 \mathrm{~m}$, lat. \\
\hline $\begin{array}{l}\text { 5G-based } \\
\text { Localisation } \\
\text { [65] }\end{array}$ & $\begin{array}{l}5 \mathrm{G} \text { communication } \\
\text { device }\end{array}$ & $\begin{array}{l}99 \% \text { below } 0.2 \mathrm{~m} \text { at } \\
100 \mathrm{MHz}\end{array}$ \\
\hline
\end{tabular}

\section{CONCLUDING REMARKS}

In this paper, a concise survey of the current state-of-the-art of vehicle localisation techniques using on-board sensing systems and their combinations with V2V and V2I systems was provided along with potentials and limitations of each technique on the implementation on autonomous vehicles. It was shown that, from the performance point of view, the LiDAR techniques show the greatest promise for the localisation of autonomous applications; however, the high power and processing requirements and its high cost render it unfeasible from cost-efficiency and commercialisation point of view. Therefore, further optimisation of LiDAR technology or alternative approaches such as localising ground penetrating Radar or vision-based localisation within LiDAR maps could offer a path towards commercially feasible systems. However, further field testing to validate the robustness of these systems, validating their performance under a variety of driving conditions, and refining operation parameters will be necessary before mass production.

It was also highlighted that integrating V2V information with on-board sensory information has great potential to improve the accuracy, robustness, and reliability of senor-based localisation systems. The main advantages of the cooperative systems are their capability of collecting information out of line-of-sight and wider range, robustness to environmental conditions, and low cost. However, V2V techniques are limited by the shared position estimates and the number of connected vehicles in the area. Infrastructure with known position can be used to share accurate position estimates and with well-planned implementation, an adequate number of position estimates can always be available. However, many of the current cooperative systems have been evaluated through simulation, and while this is useful for the feasibility study and initial performance evaluation, a variety of field tests will be required under different possible driving and communication conditions before these systems are ready to be deployed. Moreover, the quality of service in V2V and V2I networks must be optimised for all possible operating conditions to ensure efficient and effective operation once deployed. The large scale of these networks means that the effect of characteristics such as transmission power, packet transmission frequency, data rate, RSU configuration, and network size must be studied and optimised to minimise latency and packet loss. Furthermore, the security of these networks must be considered to ensure the safety and privacy of all communication over the network, as well as the effect of any security mechanisms to the performance of the network. Also, the effect of network failure on the performance of the autonomous vehicles must be considered. Furthermore, the emergent $5 \mathrm{G}$ communication systems could facilitate secure, reliable, and ultra-low latency communications among vehicles and with infrastructure, assisting cooperative localisation techniques suitable for autonomous driving.

The environment in which the solution is to be deployed must be considered as well. As previously discussed, each of the sensors and localisation techniques have distinct pros and cons. Therefore, the best solution may depend on environmental factors such as weather, traffic or road conditions. For instance, camera based solutions which depend on road marking detection would not be feasible in parts of the world where road markings are covered e.g. by snow most of the year or on roads which lack these markings completely. Similarly, V2X solutions rely heavily on dense networks of vehicles and/or infrastructure. While dense infrastructure deployment and a large number of nearby vehicles may be a reasonable assumption for example in urban or highway environments, this might not be the case for rural environments. Also, techniques which rely on GPS have to account for environments where GPS signals could be completely unavailable, such as tunnels. In such environments V2I solutions such as VLC may be required to provide reliable localisation.

Further research will be required to validate and refine the performance and robustness of the most promising localisation techniques. Moreover, the integration of cooperative techniques with on-board sensing system will need further research to find the optimal way to fuse these localisation techniques together for increased reliability and accuracy. Furthermore, the optimal configuration of Vehicular Ad Hoc Networks will need to be investigated to achieve low packet loss and latency in highly congested networks with high-speed nodes and varying topology. Additionally, the reliability of localisation systems needs to be improved before commercialisation, including consideration of sensor failures and fault tolerant systems.

\section{APPENDIX A: DEFINITIONS}

Here is a list of definitions of terms used in this report.

- Accuracy: How close the measured position or value is from the systems true position or value.

- Dead Reckoning: A localisation method using an initial known position and odometry to calculate the changing position during movement.

- Error: The difference between an estimated value or position to the true value or position.

- Feature map: An information layer which includes the environment features (e.g. road marks, buildings, obstacles, other road users, etc.) and their locations.

- IEEE 802.11p: An addition to the IEEE 802.11 standard to include Wireless Access in Vehicular Environments (WAVE) communication system and defines 
modifications required to support it. IEEE 802.11 standard defines the specifications for $\mathrm{Wi}-\mathrm{Fi}$ products.

- Internet of Things: A network of interconnected technologies, devices, objects, and services. See [5] for further details.

- Odometry: The measure of change in position over time, relative to the starting position

- Packet Loss: The loss of packets, units of information transferred over networks, as they fail to reach their destination. Typically caused by network congestion.

- Robustness: Ability of the system to cope with errors and erroneous inputs.

- VANET: Vehicular Ad Hoc Networks are a type of mobile ad hoc network (MANET), in which each node is free to move independently and will therefore frequently change which nodes they are connected to. VANETs are expected to use the IEEE 802.11p standard for wireless communication.

APPENDIX B: LIST OF ABBREVIATIONS

\begin{tabular}{|c|c|}
\hline Abbreviation & Description \\
\hline $3 D$ & Three Dimensional \\
\hline $5 G$ & Fifth Generation \\
\hline$A G P S$ & Assisted GPS \\
\hline$A O A$ & Angle-of-Arrival \\
\hline$C A M$ & Cooperative Awareness Message \\
\hline COVEL & $\begin{array}{l}\text { Cooperative Vehicle Localisation for } \\
\text { Efficient Urban Mobility }\end{array}$ \\
\hline$D E N M$ & $\begin{array}{l}\text { Decentralised Environmental Notification } \\
\text { Message }\end{array}$ \\
\hline$D G P S$ & Differential Global Positioning System \\
\hline$D S R C$ & Dedicated Short Range Communication \\
\hline$E C E F$ & Earth Centred Earth Fixed \\
\hline$E C I$ & Earth-Centred Inertial \\
\hline$E K F$ & Extended Kalman Filter \\
\hline$F M C W$ & Frequency Modulation Continuous Wave \\
\hline$G I S$ & Geographical Information System \\
\hline GNSS & Global Navigation Satellite Systems \\
\hline GPS & Global Positioning System \\
\hline$G S M$ & Global System for Mobile \\
\hline
\end{tabular}

Communications

High Definition

$H D$

Human-Machine Interface

$H M I$

Institute of Electrical and Electronics

IEEE Engineers

IMU Inertial Measurement Unit

IOT Internet of Things

IOV Internet of Vehicles

$I R-U W B$

Impulse Radio Ultrawide Bandwidth

ITS Intelligent Transportation System

KF Kalman Filter

LDM Local Dynamic Map

$L E D \quad$ Light-Emitting Diode

LGPR Localising Ground Penetrating Radar

LiDAR Light Detection and Ranging

LRF Laser Range Finder

MAC Media Access Control

MAFS Master Antenna Fixed Stations

MANET

Mobile Ad Hoc Network

MSE

PF

PHY

Radar

RANSAC

Mean Square Error

RFID

$R M S$

Particle Filter

Physical Layer

Radio detecting and Ranging

Random Sample Consensus

Radio Frequency Identification

Root Mean Square

RSS Radio Signal Strength

$R S U$

Road Side Unit

$R T K$

$S A M$

Real Time Kinematic

SLAM

SNR
Service Announcement Message

Simultaneous Localisation and Mapping

Signal-to-Noise-Ratio 


$\begin{array}{cl}\text { SRR } & \text { Short Range Radar } \\ \text { TDOA } & \text { Time-Difference-of-Arrival } \\ \text { TOA } & \text { Time-of-Arrival } \\ \text { TTFF } & \text { Time-To-First-Fix } \\ \text { UHF } & \text { Ultra-High Frequency } \\ \text { UWB } & \text { Ultra-Wide Band } \\ V 2 I & \text { Vehicle-to-Infrastructure } \\ V 2 V & \text { Vehicle-to-Vehicle } \\ V 2 X & \text { Vehicle-to-Everything } \\ V A N E T & \text { Vehicular Ad Hoc Network } \\ V L C & \text { Visible Light Communication } \\ W A V E & \text { Wireless Access in Vehicular Environment }\end{array}$

\section{REFERENCES}

[1] Bureau of Transportation Statistics, "Table 1-11: Number of U.S. Aircraft, Vehicles, Vessels, and Other Conveyances," 2016. [Online]. Available: http://www.rita.dot.gov/bts/sites/rita.dot.gov.bt s/files/publications/national_transportation_stati stics/html/table_01_11.html. [Accessed 1 December 2016].

[2] Department for Transport, "Research on the Impacts of Connected and Autonomous Vehicles (CAVs) on Traffic Flow: Summary Report," 52016. [Online].

Available: https://www.gov.uk/government/uploads/syste m/uploads/attachment_data/file/530091/impact s-of-connected-and-autonomous-vehicles-on-traf fic-flow-summary-report.pdf. [Accessed 2903 2017].

[3] K. Jo, J. Kim, D. Kim, C. Jang and M. Sunwoo, "Development of Autonomous Car - Part I: Distributed System Architecture and Development Process," IEEE Transactions on Industrial Electronics, vol. 61, no. 10, pp. 7131-7140, 2014.

[4] 5G-PPP, "5G Automotive Vision," 2015. [Online]. Available: https://5g-ppp.eu/wp-content/uploads/2014/02/ 5G-PPP-White-Paper-on-Automotive-Vertical-Sect ors.pdf. [Accessed 122 2017].

[5] R. Minerva, A. Biru and D. Rotondi, "Towards a definition of the Internet of Things (IoT)," 2015. [Online]. Available: https://iot.ieee.org/images/files/pdf/IEEE_IoT_To wards_Definition_Internet_of_Things_Revision1_ 27MAY15.pdf.

[6] Y. Fangchun, W. Shangguang, L. Jinglin, L. Zhihan and S. Qibo, "An overview of Internet of Vehicles," China Communications, vol. 11, no. 10, pp. 1-15, 2014.

[7] Asia-Pacific Economic Cooperation (APEC), "White Paper of Internet of Vehicles," in 50th Telecommunications and Information Working Group Meeting, Brisbane, Australia, 2014.

[8] Y. Cao and N. Wang, "Towards Efficient Electric Vehicle Charging Using VANET-Based Information Dissemination," IEEE Transactions on Vehicular Technology, vol. 66, no. 4, pp. 2886-2901, 2017.

[9] Y. Cao, S. Yang, G. Min, X. Zhang, H. Song, O. Kaiwartya and N. Aslam, "A Cost-Efficient Communication Framework or Battery Switch Based Electric Vehicle Charging," IEEE Communications Magazine, vol. 5, no. 5, pp. 162-169, 2017.

[10] N. Lu, N. Cheng, N. Zhang, X. Shen and J. W. Mark, "Connected Vehicles: Solutions and Challenges," IEEE Internet of Things Journal, vol. 1, no. 4, pp. 289-299, 2014.

[11] Mobileye, "The Mapping Challenge," 2017. [Online]. Available: http://www.mobileye.com/our-technology/rem/. [Accessed 0329 2017].

[12] NVIDIA, "Introducing the new nvidia drive px2," 2017. [Online]. Available: http://www.nvidia.com/object/drive-px.html. [Accessed April 20 2017].

[13] OpenStreetMap, "Micromapping," 2016. [Online]. 
Available:

http://wiki.openstreetmap.org/wiki/Micromappi ng. [Accessed 2903 2017].

[14] ISO, "ISO/TS 18750:2015, Intelligent transport systems -- Cooperative systems -- Definition of a global concept for Local Dynamic Maps," 2015. [Online].

Available:

https://www.iso.org/standard/63273.html.

[Accessed 2903 2017].

[15] ETSI, "Intelligent Transport Systems (ITS); Vehicular Communications; Basic Set of Applications; Local Dynamic Map (LDM)," 2014. [Online]. Available: http://www.etsi.org/deliver/etsi_en/302800_302 899/302895/01.00.00_20/en_302895v010000a.p df. [Accessed 2903 2017].

[16] N. Mattern and G. Wanielik, "Vehicle Localization in Urban Environments using Feature Maps and Aerial Images," in Intelligent Transportation Systems (ITSC), 2011 14th International IEEE Conference on, Washington, DC, 2011.

[17] G. Vivo, "SAFESPOT Core Architecture - LDM API and Usage Reference," 2010. [Online]. Available: http://www.safespot-eu.org/documents/SF_D7.3 .1_Annex2_LDM_API_and_Usage_Reference_v0. 7.pdf.

[18] F. Nelli, “3D Laser Scanning," 2016. [Online]. Available:

http://www.meccanismocomplesso.org/en/laserscanning-3d/. [Accessed 2903 2017].

[19] I. Skog and P. Händel, "In-Car Positioning and Navigation Technologies - A Survey," IEEE Transactions on Intelligent Transportation Systems, vol. 10, no. 1, pp. 4-21, 2009.

[20] H. Tan and J. Huang, "DGPS-Based Vehicle-to-Vehicle Cooperative Collision Warning: Engineering Feasibility Viewpoints," IEEE Transactions on Intelligent Transportation Systems, vol. 7, no. 4, pp. 415-428, 2006.

[21] J. Levinson, M. Montemerlo and S. Thrun, "Map-Based Precision Vehicle Localization in
Urban Environments," Robotics: Science and Systems, vol. 3, pp. 121-128, 2007.

[22] G. Djuknic and R. Richton, "Geolocation and Assisted GPS," Computer, vol. 34, no. 2, pp. 123-125, 2001.

[23] European Commission, "Galileo," 2017. [Online]. Available:

https://ec.europa.eu/growth/sectors/space/galile o_en. [Accessed 153 2017].

[24] European Commission, "Satellite Navigation Why Galileo?," 2011. [Online]. Available: https://ec.europa.eu/growth/sectors/space/galile o_en. [Accessed 153 2017].

[25] NavStar, "NavStar GPS User Equipment Introduction," 1996. [Online]. Available: https://www.navcen.uscg.gov/pubs/gps/gpsuser/ gpsuser.pdf. [Accessed 16 May 2017].

[26] F. Zhang, H. Stähle, G. Chen, C. Chen, C. Simon, C. Buckl and A. Knoll, "A Sensor Fusion Approach for Localization with Cumulative Error Elimination," Multisensor Fusion and Integration for Intelligent Systems (MFI), 2012 IEEE Conference on, pp. 1-6, 2012.

[27] C. Li, B. Dai and T. Wu, "Vision-based Precision Vehicle Localization In Urban Environments," in Chinese Automation Congress (CAC), Changsha, China, 2013.

[28] I. Parra, M. Sotelo, D. Llorca and M. Ocaña, "Robust visual odometry for vehicle localization in urban environments," Robotica, vol. 28, pp. 441-452, 2010.

[29] S. Kamijo, Y. Gu and L. Hsu, "Autonomous Vehicle Technologies: Localization and Mapping," IEICE Fundamentals Review, vol. 9, no. 2, pp. pp. 131-141, 2015.

[30] J. Suhr, J. Jang, D. Min and H. Jung, "Sensor Fusion-Based Low-Cost Vehicle Localization System for Complex Urban Environments," IEEE Transactions on Intelligent Transportation Systems, vol. 18, no. 5, pp. 1078-1086, 2017. 
[31] E. Ward and J. Folkesson, "Vehicle localization with low cost radar sensors," Intelligent Vehicles Symposium (IV), 2016, IEEE, 2016.

[32] D. Vivet, F. Gérossier, P. Checchin, L. Trassoudaine and R. Chapuis, "Mobile Ground-Based Radar Sensor for Localization and Mapping: An Evaluation of two Approaches," International Journal of Advanced Robotic Systems, vol. 10, no. 5, pp. 307-318, 2013.

[33] M. Cornick, J. Koechling, B. Stanley and B. Zhang, "Localizing Ground Penetrating RADAR: A Step Toward Robust Autonomous Ground Vehicle Localization," Journal of Field Robotics, vol. 33, no. 1, pp. 82-102, 2016.

[34] Velodyne LiDAR, "Product Guide," 2016. [Online]. Available:

http://velodynelidar.com/docs/datasheet/LiDAR \%20Comparison\%20chart_Rev-A_Web.pdf.

[Accessed 2811 2016].

[35] A. Hata and D. Wolf, "Feature Detection for Vehicle Localization in Urban Environments Using Multilayer LIDAR," IEEE Transactions On Intelligent Transportation Systems, vol. 17, no. 2, pp. 420-429, 2016.

[36] J. Levinson and S. Thrun, "Robust Vehicle Localization in Urban Environments Using Probabilistic Maps," Robotics and Automation (ICRA), 2010 IEEE International Conference on, pp. 4372-4378, 2010.

[37] J. Castorena and S. Agarwal, "Ground-Edge-Based LIDAR Localization Without a Reflectivity Calibration for Autonomous Driving," IEEE Robotics and Automation Letter, vol. 3, no. 1, pp. 344-351, 2018.

[38] H. Kim, B. Liu, C.-Y. Goh, S. Lee and H. Myung, "Robust Vehicle Localization Using Entropy-Weighted Particle Filter-based Data Fusion of Vertical and Road Intensity Information for a Large Scale Urban Area," IEEE Robotics and Automation Letters, vol. 2, no. 3, pp. 1518-1524, 2017.
[39] R. W. Wolcott and R. M. Eustice, "Robust LIDAR localization using multiresolution Gaussian mixture maps for autonomous driving," The International Journal of Robotics Research, vol. 36, no. 3, pp. 292-319, 2017.

[40] R. Wolcott and R. Eustice, "Visual Localization within LIDAR maps for automated urban driving," IEEE International Conference on Intelligent Robots and Systems, pp. 176-183, 2014.

[41] L. Wei, C. Cappelle and Y. Ruichek, "Horizontal/Vertical LRFs and GIS Maps Aided Vehicle Localization in Urban Environment," in IEEE Annual Conference on Intelligent Transportation Systems (ITSC 2013), The Hague, 2013.

[42] S. Jung, J. Kim and S. Kim, "Simultaneous localization and mapping of a wheel-based autonomous vehicle with ultrasonic sensors," Artificial Life and Robotics, vol. 14, no. 2, pp. 186-190, 2009.

[43] M. Obst, N. Mattern, R. Schubert and G. Wanielik, "Car-to-Car Communication for Accurate Vehicle Localization - the CoVeL Approach," 9th International Multi-Conference on Systems, Signals and Devices, 2012.

[44] K. Cheok, G. Smid, G. Hudas and J. Overholt, "An Ultra-Wideband RF Method for Localizing an Autonomous Mobile Robot," Jadi Inc., Troy, 2004.

[45] M. Terré, M. Pischella and E. Vivier, "UWB," in Wireless Telecommunication Systems, London, ISTE, 2013, pp. 155-163.

[46] F. Nekoogar, Ultra-Wideband Communications: Fundamentals and Applications, 1st ed., New Jersey: Prentice Hall, 2006.

[47] J. Ibanez-Guzman, S. Lefevre, A. Mokkadem and S. Rodhaim, "Vehicle to Vehicle communications applied to Road Intersection Safety, Field Results," in 13th International IEEE Annual Conference on Intelligent Transportation Systems, 2010. 
[48] P. Papadimitratos, A. de La Fortelle, K. Evenssen, R. Brignolo and S. Cosenza, "Vehicular Communication Systems: Enabling technologies, applications, and future outlook on Intelligent Transportation," IEEE Communications Magazine, vol. 47, no. 11, 2009.

[49] ETSI, “ETSI EN 302 637-2; Intelligent Transport Systems (ITS); Vehicular communications; Basic set of Applications; Part 2: Specification of Cooperative Awareness Basic Service," 2014. [Online]. Available: http://www.etsi.org/deliver/etsi_en/302600_302 699/30263702/01.03.02_60/en_30263702v01030 2p.pdf.

[50] ETSI, “ETSI EN 302 637-3: Intelligent Transport Systems (ITS); Vehicular Communications; Basic Set of Applications; Part 3: Specifications of Decentralized Environmental Notification Basic Service," 2014. [Online]. Available: http://www.etsi.org/deliver/etsi_en/302600_302 699/30263703/01.02.01_30/en_30263703v01020 $1 \mathrm{v} . \mathrm{pdf}$.

[51] M. Sepulcre, J. Gozalvez and J. Hernandez, "Cooperative vehicle-to-vehicle active safety testing under challenging conditions," Transportation Research Part C, vol. 26, pp. 233-255, 2013.

[52] S. Fujii, A. Fujita, T. Umedu, H. Yamaguchi, T. Higashino, S. Kaneda and M. Takai, "Cooperative Vehicle Positioning via V2V Communications and Onboard Sensors," Vehicular Technology Conference, 2011.

[53] M. Rohani, D. Gingras and D. Gruyer, "A New Decentralized Bayesian Approach for Cooperative Vehicle Localization Based on Fusion of GPS and VANET Based Inter-Vehicle Distance Measurement," IEEE Intelligent Transport Systems Magazine, vol. 7, no. 2, pp. 85-95, 2015.

[54] N. Mattern, M. Obst, R. Schubert and G. Wanielik, "Co-operative Vehicle Localization Algorithm Evaluation of the COVEL Approach," 9th International Multi-Conference on Systems,
Signals and Devices, 2012.

[55] R. Ordóñez-Hurtado, W. Griggs, E. Crisostomi and R. Shorten, "Cooperative Positioning in Vehicular Ad-hoc Networks Supported by Stationary Vehicles," IEEE Transactions on Intelligent Transportation Systems and Magazine, 2015.

[56] K. Golestan, S. Seifzadeh, M. Kamel, F. Karray and F. Sattar, "Vehicle Localization in VANETs Using Data Fusion and V2V Communication," Proceedings of the second ACM international symposium on design and analysis of intelligent vehicular networks and application, 2012.

[57] F. Ahammed, J. Taheri, A. Zomaya and M. Ott, "VLOCI: Using Distance Measurements to Improve the Accuracy of Location Coordinates in GPS-Equipped VANETs," Lecture Notes of the Institute for Computer Sciences, Social-Informatics and Telecommunications Engineering, LNICST, vol. 73, pp. 149-161, 2012.

[58] L. Altoaimy and I. Mahgoub, "Fuzzy Logic Based Localization for Vehicular Ad Hoc Networks," IEEE, Symposium on Computational Intelligence in Vehicles and Transportation Systems (CIVTS), 2014.

[59] A. Boukerche, H. Oliveira, E. Nakamura and A. Loureiro, "Vehicular Ad Hoc Networks: A New Challenge for Localization-Based Systems," Computer Communications, vol. 31, pp. 2838-2849, 2008.

[60] J. Vales-Alonso, F. Vicente-Carrasco and J. Alcaraz, "Optimal configuration of roadside beacons in V2I communication," Computer Networks, vol. 55, pp. 3142-3153, 2011.

[61] O. Hassan, I. Adly and K. Shehata, "Vehicle Localization System based on IR-UWB for V2I Applications," in Computer Engineering \& Systems (ICCES), 2013 8th International Conference on, Cairo, Egypt, 2013.

[62] A. Fascista, G. Ciccarese, A. Coluccia and G. Ricci, "A localization algorithm based on V2I communications and AOA estimation," IEEE 
Signal Processing Letters, no. 99, 2016.

[63] A. Khattab, Y. Fahmy and A. Wahab, "High Accuracy GPS-Free Vehicle Localization Framework via an INS-Assisted Single RSU," International Journal of Distributed Sensor Networks, vol. 11, no. 5, 2015.

[64] N. Houdali, T. Ditchi, E. Gérome, J. Lucas and S. Holé, "RF Infrastructure Cooperative Systems for in Lane Vehicle Localization," Electronics, vol. 3, no. 4, pp. 598-608, 2014.

[65] J. del Peral-Rosado, J. López-Salcedo, S. Kim and G. Seco-Granados, "Feasibility Study of 5G-based Localization for Assisted Driving," IEEE, 2016 International Conference on Localization and GNSS (ICL-GNSS), 2016.

[66] S. Ergen, H. Tetikol, M. Kontik, R. Sevlian, R. Rajagopal and P. Varaiya, "RSSI-Fingerprinting-Based Mobile Phone Localization with Route Constraints," IEEE Transactions on Vehicular Technology, vol. 63, no. 1, pp. 423-428, 2014.

[67] A. Varshavsky, M. Chen, E. de Lara, J. Froehlich, D. Haehnel, J. Hightower, A. LaMarca, F. Potter, S. T., K. Tang and I. Smith, "Are GSM phones the solution for localization?," In Mobile Computing Systems and Applications, Proceedings of the 7th IEEE Workshop on, 2006, pp. 20-28, 2006.

[68] M. Chen, D. Haehnel, J. Hightower, T. Sohn, A. LaMarca, I. Smith, D. Chmelev, J. Hughes and F. Potter, "Practical metropolitan-scale positioning for gsm phones," In: Proceedings of the 8th Ubicomb, Orange County, California, 2006, pp. 225-242, 2006.

[69] C. Chen, C. Lee and C. Lo, "Vehicle Localization and Velocity Estimation Based on Mobile Phone Sensing," IEEE Access, vol. 4, pp. 803-817, 2016.

[70] H. Xue, H. Zhu, S. Cao, S. Chang and J. Cao, "UPS: Combatting Urban Vehicle Localization with Cellular-Aware Trajectories," In: Global Communications Conference (GLOBECOM), 2016.
[71] L. Bento, R. Parafita and U. Nunes, "Inter-Vehicle Sensor Fusion for Accurate Vehicle Localization Supported by V2V and V2I Communications," in Intelligent Transportation Systems (ITSC), 2012 15th International IEEE Conference on, Anchorage, Alaska, 2012.

[72] L. Bento, R. Chelim and U. Nunes, "Collaborative Vehicle Self-Localization using Multi-GNSS Receivers and V2V/V2I Communications," in Intelligent Transportation Systems (ITSC), 2015 IEEE 18th International Conference on, IEEE, 2015.

[73] H. Qin, Y. Peng and W. Zhang, "Vehicles on RFID: Error-Cognitive Vehicle Localization in GPS-Less Environments," IEEE Transactions on Vehicular Technology, vol. 66, no. 11, pp. 9943-9957, 2017.

[74] S. Arai, S. Mase, T. Yamazato, T. Endo, T. Fujii, M. Tanimoto, K. Kidono, Y. Kimura and Y. Ninomiya, "Experimental on hierarchical transmission scheme for visible light communication using LED traffic light and high-speed camera," in IEEE 66th Vehicular Technology Conference (VTC-2007 Fall), Baltimore, MD, USA, 2007.

[75] S. Arai, Y. Shiraki, T. Yamazato, H. Okada, T. Fujii and T. Yendo, "Multiple LED arrays acquisition for image-sensor-based I2V-VLC using block matching," in IEEE 11th Consumer Communications and Networking Conference (CCNC), Las Vegas, NV, USA, 2014.

[76] C. Li and S. Shimamoto, "An open traffic light control model for reducing vehicles $\mathrm{CO} 2$ emissions based on ETC vehicles," IEEE Transactions on Vehicular Technology, vol. 61, no. 1, pp. 97-110, 2012.

[77] C. B. Liu, B. Sadeghi and E. W. Knightly, "Enabling Vehicular Visible Light Communication (V2LC) networks," in Proceedings of the 8th ACM International Workshop on vehicular inter-networking, Las Vegas, NV, USA, 2011.

[78] L.-C. Wu and H.-M. Tsai, "Modeling vehicle-to-vehicle visible light communication link duration with empirical data," in Proceedings of IEEE Globecom Workshops, Atlanta, GA, USA, 
2013.

[79] S.-H. You, S.-H. Chang, H.-M. Lin and H.-M. Tsai, "Visible light communications for scooter safety," in Proceedings of MobiSys '13, Taipei, 2013.

[80] S.-H. Yu, O. Shih, H.-M. Tsai, N. Wisitpongphan and R. D. Roberts, "Smart automotive lighting for vehicle safety," IEEE Communications Magazine, vol. 51, no. 12, pp. 50-59, 2013.

[81] R. Roberts, P. Gopalakrishnan and S. Rathi, "Visible Light Positioning: Automotive Use Case," in IEEE Vehicular Networking Conference (VNC), Jersey City, NJ, USA, 2010.

[82] S. Rajagopal, R. D. Roberts and S.-K. Lim, "IEEE 802.15.7 visible light communication: modulation schemes and dimming support," IEEE Communications Magazine, vol. 50, no. 3, pp. 72-82, 2012.

[83] P. H. Pathak, X. Feng, P. Hu and P. Mohapatra, "Visible Light Communication, Networking, and Sensing: A Survey, Potential and Challenges," IEEE Communications Surveys \& Tutorials, vol. 17, no. 4, pp. 2047-2077, 2015.

[84] L. Li, C. Peng and F. Zhai, "Epsilon: A Visible Light Based Positioning System," in Proceedings of the 11th USENIX Symposium on Networked Systems Design and Implementation (NSDI '14), Seattle,
[85]

B. W. Kim and S.-Y. Jung, "Vehicle Positioning Scheme Using V2V and V2I Visible Light Communications," in IEEE 83rd Vehicular Technology Conference (VTC Spring), Nanjing, China, 2016.

[86] R. Schmidt, T. Köllmer, T. Leinmüller, B. Böddeker and G. Schäfer, "Degradation of Transmission Range in VANETs caused by Interference," PIK Praxis Der Informationsverarbeitung Und Kommunikation, vol. 32, no. 4, pp. 224-234, 2009.

[87] A. Fonseca and T. Vazão, "Applicability of position-based routing for VANET in highways and urban environment," Journal of Network and Computer Applications, vol. 36, pp. 961-973, 2013.

[88] R. Engoulou, M. Bellaïche, S. Pierre and A. Quintero, "VANET Security Surveys," Computer Communications, vol. 44, no. 5, pp. 1-13, 2014.

\begin{tabular}{|c|c|c|c|c|}
\hline $\begin{array}{l}\text { Technique } \\
\text { (Reference) }\end{array}$ & Sensors & Accuracy & Advantages & Disadvantages \\
\hline Pure GPS & GPS & $\sim 10 \mathrm{~m}$ & Low cost & $\begin{array}{l}\text { Low accuracy } \\
\text { Poor signal availability }\end{array}$ \\
\hline $\begin{array}{l}\text { GPS/IMU in ECEF } \\
\text { coordinates } \\
\text { [26] }\end{array}$ & GPS \& IMU & $\begin{array}{l}7.2 \mathrm{~m} \\
\text { (RMSE) }\end{array}$ & $\begin{array}{l}\text { Low cost } \\
\text { IMU provides positioning } \\
\text { during GPS signal blockage }\end{array}$ & $\begin{array}{l}\text { Low accuracy } \\
\text { Cumulative errors }\end{array}$ \\
\hline $\begin{array}{l}\text { Two-stage vision-based } \\
\text { SLAM } \\
\text { [27] }\end{array}$ & Camera & $\begin{array}{l}0.75 \mathrm{~m} \\
\text { (Mean) }\end{array}$ & Low cost & $\begin{array}{l}\text { Susceptible to illumination and } \\
\text { observation angle }\end{array}$ \\
\hline $\begin{array}{l}\text { Stereovision odometry } \\
\text { [28] }\end{array}$ & Camera & $\begin{array}{l}\text { Up to } 20.5 \mathrm{~m} \text { cumulative } \\
\text { error over } 166 \mathrm{~m} \text { distance }\end{array}$ & Low Cost & $\begin{array}{l}\text { Low accuracy } \\
\text { Cumulative errors }\end{array}$ \\
\hline $\begin{array}{l}\text { Vision-based localisation } \\
\text { with lane detection } \\
\text { [29] }\end{array}$ & Camera, GPS, IMU & $\begin{array}{l}0.73 m \\
\text { (Mean) }\end{array}$ & Low cost & $\begin{array}{l}\text { Susceptible to illumination and } \\
\text { observation angle }\end{array}$ \\
\hline $\begin{array}{l}\text { Vision-based localisation } \\
\text { with road marker } \\
\text { detection } \\
\text { [30] }\end{array}$ & Camera, GPS, IMU & $\begin{array}{l}0.58 \mathrm{~m} \text {, lat. } \\
1.43 \mathrm{~m}, \text { long. } \\
\text { (Mean) }\end{array}$ & Low cost & $\begin{array}{l}\text { Susceptible to illumination and } \\
\text { observation angle }\end{array}$ \\
\hline $\begin{array}{l}\text { Aerial Image-based } \\
\text { localisation } \\
\text { [16] }\end{array}$ & Camera, GPS, IMU & $80 \%$ within $1 \mathrm{~m}$ & Low cost & High errors \\
\hline
\end{tabular}




\begin{tabular}{|c|c|c|c|c|}
\hline $\begin{array}{l}\text { Microwave-Radar SLAM } \\
\text { [32] }\end{array}$ & Microwave Radar & $\begin{array}{l}10.5 \mathrm{~m} \\
\text { (Mean) }\end{array}$ & $\begin{array}{l}\text { Low power requirements } \\
\text { Low cost }\end{array}$ & Low accuracy \\
\hline $\begin{array}{l}\text { Short Range Radar } \\
\text { SLAM } \\
\text { [31] }\end{array}$ & Radar, GPS, IMU & $\begin{array}{l}0.07 \mathrm{~m} \text {, lat. } \\
0.38 \mathrm{~m}, \text { long. } \\
\text { (RMSE) }\end{array}$ & $\begin{array}{l}\text { Low power requirements } \\
\text { Low cost } \\
\text { High accuracy }\end{array}$ & $\begin{array}{l}\text { Low robustness to dynamic } \\
\text { environments }\end{array}$ \\
\hline $\begin{array}{l}\text { Localising Ground } \\
\text { Penetrating Radar } \\
\text { [33] }\end{array}$ & LGPR, GPS, IMU & $\begin{array}{l}0.04 \mathrm{~m} \\
\text { (RMSE) }\end{array}$ & $\begin{array}{l}\text { Very high accuracy } \\
\text { Robust to weather and } \\
\text { illumination conditions }\end{array}$ & $\begin{array}{l}\text { Lack of testing } \\
\text { Sensitivity (e.g. to frost heave, } \\
\text { thaw settlement) uncertain }\end{array}$ \\
\hline $\begin{array}{l}\text { LiDAR SLAM } \\
\text { [35], [21], [36], [37], } \\
\text { [38], [39] }\end{array}$ & LiDAR, GPS, IMU & $\begin{array}{l}0.017 \mathrm{~m} \text {, lat. } \\
0.033 \mathrm{~m} \text {, long. } \\
\text { (RMSE) }\end{array}$ & $\begin{array}{l}\text { High accuracy } \\
\text { Robust to changes in } \\
\text { environment }\end{array}$ & $\begin{array}{l}\text { High cost } \\
\text { High power \& processing } \\
\text { requirements } \\
\text { Sensitive to weather conditions }\end{array}$ \\
\hline $\begin{array}{l}\text { Camera localisation } \\
\text { within LiDAR map } \\
\text { [40] }\end{array}$ & Camera, IMU & $\begin{array}{l}0.14 \mathrm{~m}, \text { lat. } \\
0.19 \mathrm{~m}, \text { long. } \\
\text { (RMSE) }\end{array}$ & $\begin{array}{l}\text { High accuracy } \\
\text { Low cost }\end{array}$ & $\begin{array}{l}\text { Requires environments to be } \\
\text { mapped using a dedicated } \\
\text { LiDAR vehicle } \\
\text { Robustness }\end{array}$ \\
\hline $\begin{array}{l}\text { LRF based localisation } \\
\text { [41] }\end{array}$ & GPS, IMU, LRF & $\begin{array}{l}3.098 \mathrm{~m} \\
\text { (Mean) }\end{array}$ & Low cost & High errors \\
\hline $\begin{array}{l}\text { Ultrasonic SLAM } \\
\text { [42] }\end{array}$ & Ultrasonic & (Not given) & $\begin{array}{l}\text { Low power requirements } \\
\text { Low cost }\end{array}$ & $\begin{array}{l}\text { Low accuracy } \\
\text { Long processing time }\end{array}$ \\
\hline
\end{tabular}

Table 2. Summary of V2V Localisation Methods.

\begin{tabular}{|c|c|c|c|c|c|}
\hline $\begin{array}{l}\text { Method } \\
\text { (Reference) }\end{array}$ & Sensors & No. of vehicles & Accuracy & Advantages & Disadvantages \\
\hline $\begin{array}{l}\text { VANET Multilateration } \\
\text { [53] }\end{array}$ & $\begin{array}{l}\text { GPS, V2V } \\
\text { communication }\end{array}$ & 5 & 3.30m (Mean) & $\begin{array}{l}\text { Low Cost } \\
\text { Does not rely on all } \\
\text { vehicles being able to } \\
\text { communicate }\end{array}$ & High Error \\
\hline $\begin{array}{l}\text { V2V and on-board } \\
\text { sensor localisation } \\
\text { [52] }\end{array}$ & $\begin{array}{l}\text { GPS, V2V } \\
\text { communication, } \\
\text { ranging sensors }\end{array}$ & $\begin{array}{l}1800 \& 1200 \\
\text { vehicles per hour } \\
\text { on } 1 \mathrm{~km} \text { of road }\end{array}$ & $0.60 m$ (Mean) & $\begin{array}{l}\text { Does not rely on all } \\
\text { vehicles being able to } \\
\text { communicate }\end{array}$ & $\begin{array}{l}\text { Requires on-board } \\
\text { ranging sensors }\end{array}$ \\
\hline $\begin{array}{l}\text { COVEL approach } \\
\text { [54] }\end{array}$ & $\begin{array}{l}\text { GPS, odometry, } \\
\text { V2V } \\
\text { communication }\end{array}$ & 6 & $\begin{array}{l}50 \% \text { within } \\
1.09 \mathrm{~m}\end{array}$ & Low Cost & $\begin{array}{l}\text { Assumes all vehicles } \\
\text { equipped with GPS } \\
\text { and V2V }\end{array}$ \\
\hline $\begin{array}{l}\text { VANET supported by } \\
\text { stationary vehicles } \\
\text { [55] }\end{array}$ & $\begin{array}{l}\text { GPS, V2V } \\
\text { communication }\end{array}$ & $20 \& 900$ & Up to $3.14 m$ & Low Cost & $\begin{array}{l}\text { Battery use while } \\
\text { stationary } \\
\text { Dependent on number } \\
\text { of parked cars nearby }\end{array}$ \\
\hline $\begin{array}{l}\text { Multilateration with } \\
\text { shared position } \\
\text { estimates in VANET } \\
\text { [56] }\end{array}$ & $\begin{array}{l}\text { GPS, gas and } \\
\text { brake pedal and } \\
\text { steering wheel } \\
\text { sensors, V2V } \\
\text { communication }\end{array}$ & 5 & $\begin{array}{l}0.52-1.65 \mathrm{~m} \\
\text { (MSE) }\end{array}$ & $\begin{array}{l}\text { Low Cost } \\
\text { Increased information } \\
\text { sharing }\end{array}$ & $\begin{array}{l}\text { Relies on the number } \\
\text { of connected vehicles }\end{array}$ \\
\hline $\begin{array}{l}\text { Weighted V2V } \\
\text { localisation based on } \\
\text { intervehicle distance } \\
\text { [57] }\end{array}$ & $\begin{array}{l}\text { GPS, V2V } \\
\text { communication }\end{array}$ & 10 & $\begin{array}{l}2.38 \mathrm{~m} \\
\text { (Mean) }\end{array}$ & $\begin{array}{l}\text { Improved robustness } \\
\text { and accuracy }\end{array}$ & $\begin{array}{l}\text { Relies on connected } \\
\text { vehicles }\end{array}$ \\
\hline $\begin{array}{l}\text { Weighted localisation } \\
\text { based on intervehicle } \\
\text { distance and SNR } \\
\text { [58] }\end{array}$ & $\begin{array}{l}\text { GPS, V2V } \\
\text { communication }\end{array}$ & $20-200$ & $\begin{array}{l}0.25 m-0.85 m \\
\text { (Mean), based } \\
\text { on network size }\end{array}$ & $\begin{array}{l}\text { Improved robustness } \\
\text { and accuracy }\end{array}$ & $\begin{array}{l}\text { Relies on connected } \\
\text { vehicles }\end{array}$ \\
\hline
\end{tabular}

Table 3. Summary of V2I Localisation Techniques.

\begin{tabular}{|c|c|c|c|c|c|}
\hline Method & $\begin{array}{l}\text { RSU } \\
\text { spacing }\end{array}$ & Sensors & Accuracy & Advantages & Disadvantages \\
\hline $\begin{array}{l}\text { IR-UWB V2I Localisation } \\
\text { [61] }\end{array}$ & $20 \mathrm{~m}$ & UWB radio sensors & $\begin{array}{l}\text { 0-20m, depending on distance to } \\
\text { RSU }\end{array}$ & $\begin{array}{l}\text { Low transmit } \\
\text { power \& cost } \\
\text { Resistant to } \\
\text { multipath }\end{array}$ & $\begin{array}{l}\text { High error at } \\
\text { edges of a } \\
\text { network }\end{array}$ \\
\hline $\begin{array}{l}\text { AOA Estimation in } \\
\text { VANET } \\
\text { [62] }\end{array}$ & $400 m$ & $\begin{array}{l}\text { WAVE } \\
\text { communication } \\
\text { device }\end{array}$ & $\begin{array}{l}\text { 1-11m, depending on distance to } \\
\text { RSU }\end{array}$ & Low cost & $\begin{array}{l}\text { High error at } \\
\text { edges of a } \\
\text { network }\end{array}$ \\
\hline $\begin{array}{l}\text { INS Assisted single RSU } \\
\text { localisation } \\
\text { [63] }\end{array}$ & $1000 \mathrm{~m}$ & $\begin{array}{l}\text { WAVE } \\
\text { communication } \\
\text { device, IMU }\end{array}$ & $1.8 \mathrm{~m}$ (RMS) & $\begin{array}{l}\text { No GPS } \\
\text { required } \\
\text { Low Cost }\end{array}$ & Requires IMU \\
\hline $\begin{array}{l}\text { RF infrastructure } \\
\text { localisation } \\
\text { [64] }\end{array}$ & $<2 m$ & $\begin{array}{l}\text { On-board UHF } \\
\text { antenna. }\end{array}$ & Up to $0.03 m$ & $\begin{array}{l}\text { Very high } \\
\text { accuracy } \\
\text { Low Cost }\end{array}$ & $\begin{array}{l}\text { Low range } \\
\text { Not tested } \\
\text { with vehicles. }\end{array}$ \\
\hline
\end{tabular}




\begin{tabular}{|c|c|c|c|c|c|}
\hline & & & & & $\begin{array}{l}\text { Lateral } \\
\text { positioning } \\
\text { only }\end{array}$ \\
\hline $\begin{array}{l}\text { 5G-based localisation } \\
\text { [65] }\end{array}$ & $\begin{array}{l}30 \mathrm{~m} \text { for } \\
1.4 \mathrm{MHz} \text { up } \\
\text { to } 350 \mathrm{~m} \\
\text { for } \\
100 \mathrm{MHz} \text {. }\end{array}$ & $\begin{array}{l}5 \mathrm{G} \text { communication } \\
\text { device }\end{array}$ & $99 \%$ below $0.2 \mathrm{~m}$ at $100 \mathrm{MHz}$. & $\begin{array}{l}\text { High } \\
\text { accuracy }\end{array}$ & $\begin{array}{l}\text { High cost of } \\
5 \mathrm{G} \text { base } \\
\text { stations }\end{array}$ \\
\hline $\begin{array}{l}\text { Wi-Fi based } \\
\text { fingerprinting } \\
{[69]}\end{array}$ & $\begin{array}{l}25 \text { Access } \\
\text { Points per } \\
1000 \mathrm{~m}^{2}\end{array}$ & Wireless Sensor & $\begin{array}{l}18 \mathrm{~m} \\
\text { (Mean) }\end{array}$ & $\begin{array}{l}\text { Low Cost } \\
\text { Utilises } \\
\text { existing } \\
\text { infrastructure }\end{array}$ & Low accuracy \\
\hline $\begin{array}{l}\text { GSM based } \\
\text { fingerprinting with } \\
\text { trajectory estimates } \\
\text { [70] }\end{array}$ & (Not given) & $\begin{array}{l}16 \text { GSM devices, } \\
\text { GPS, IMU }\end{array}$ & $4.2 \mathrm{~m}$ (Mean) & $\begin{array}{l}\text { Low Cost } \\
\text { Utilises } \\
\text { existing } \\
\text { infrastructure }\end{array}$ & $\begin{array}{l}\text { Requires } \\
\text { frequent } \\
\text { database } \\
\text { updates }\end{array}$ \\
\hline $\begin{array}{l}\text { V2V and V2I supported } \\
\text { localisation with } \\
\text { magnetic markers } \\
\text { [71] }\end{array}$ & (Not given) & $\begin{array}{l}\text { WAVE } \\
\text { communication } \\
\text { device, GPS, IMU, } \\
\text { magnetic sensors, } \\
\text { laser scanner }\end{array}$ & $1.03 \mathrm{~m}$ (RMS) & $\begin{array}{l}\text { Error } \\
\text { slippage can } \\
\text { be corrected } \\
\text { by V2V and } \\
\text { laser } \\
\text { scanners }\end{array}$ & $\begin{array}{l}\text { Large number } \\
\text { of sensors } \\
\text { required } \\
\text { Relies on } \\
\text { magnetic } \\
\text { markers }\end{array}$ \\
\hline $\begin{array}{l}\text { GPS/V2V/V2I } \\
\text { localisation } \\
\text { [72] }\end{array}$ & (Not given) & $\begin{array}{l}\text { WAVE } \\
\text { communication } \\
\text { device, GPS }\end{array}$ & $0.698 \mathrm{~m}$ (RMS) & Low cost & $\begin{array}{l}\text { High error in } \\
\text { Roll estimates }\end{array}$ \\
\hline $\begin{array}{l}\text { RFID and V2V based } \\
\text { GPS-less localisation }\end{array}$ & $200 \mathrm{~m}$ & $\begin{array}{l}\text { RFID reader, IMU, } \\
\text { WAVE } \\
\text { communication }\end{array}$ & 0.71 (Mean) & $\begin{array}{l}\text { Low cost } \\
\text { Does not } \\
\text { require } \\
\text { dense VANET }\end{array}$ & $\begin{array}{l}\text { Reduced } \\
\text { accuracy } \\
\text { when turning }\end{array}$ \\
\hline
\end{tabular}

